\title{
SEARCH FOR PRECURSOR ERUPTIONS AMONG TYPE IIB SUPERNOVAE
}

\author{
Nora L. Strotjohann ${ }^{1,2}$, Eran O. Ofek ${ }^{1}$, Avishay Gal-Yam ${ }^{1}$, Mark Sullivan ${ }^{3}$, Shrinivas R. Kulkarni ${ }^{4}$, Nir J. Shaviv ${ }^{5,6}$,

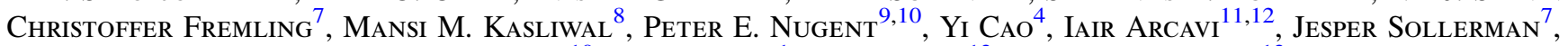 \\ Alexei V. Filippenko ${ }^{10}$, Ofer Yaron ${ }^{1}$, Russ Laher ${ }^{13}$, and Jason Surace ${ }^{13}$ \\ ${ }^{1}$ Benoziyo Center for Astrophysics, Weizmann Institute of Science, 76100 Rehovot, Israel \\ ${ }^{2}$ Desy Zeuthen, D-15738 Zeuthen, Germany \\ ${ }^{3}$ School of Physics and Astronomy, University of Southampton, Southampton SO17 1BJ, UK \\ ${ }^{4}$ Cahill Center for Astronomy and Astrophysics, California Institute of Technology, Pasadena, CA 91125, USA \\ ${ }^{5}$ School of Natural Sciences, Institute for Advanced Study, 1 Einstein Drive, Princeton, NJ 08540, USA \\ ${ }^{6}$ Racah Institute of Physics, Hebrew University, Jerusalem 91904, Israel \\ ${ }^{7}$ The Oskar Klein Centre, Department of Astronomy, Stockholm University, AlbaNova, SE-10691 Stockholm, Sweden \\ ${ }^{8}$ Observatories of the Carnegie Institution for Science, 813 Santa Barbara Street, Pasadena, CA 91101, USA \\ ${ }^{9}$ Lawrence Berkeley National Laboratory, 1 Cyclotron Road, Berkeley, CA 94720, USA \\ ${ }^{10}$ Department of Astronomy, University of California, Berkeley, CA 94720-3411, USA \\ ${ }^{11}$ Las Cumbres Observatory Global Telescope Network, 6740 Cortona Drive, Suite 102, Goleta, CA 93111, USA \\ ${ }_{12}$ Kavli Institute for Theoretical Physics, University of California, Santa Barbara, CA 93106, USA \\ ${ }^{13}$ Spitzer Science Center, California Institute of Technology, M/S 314-6, Pasadena, CA 91125, USA \\ Received 2015 June 16; accepted 2015 August 18; published 2015 September 28
}

\begin{abstract}
The progenitor stars of several Type IIb supernovae (SNe) show indications of extended hydrogen envelopes. These envelopes might be the outcome of luminous energetic pre-explosion events, so-called precursor eruptions. We use the Palomar Transient Factory (PTF) pre-explosion observations of a sample of 27 nearby SNe IIb to look for such precursors during the final years prior to the $\mathrm{SN}$ explosion. No precursors are found when combining the observations in 15-day bins, and we calculate the absolute-magnitude-dependent upper limit on the precursor rate. At the $90 \%$ confidence level, SNe IIb have on average $<0.86$ precursors as bright as an absolute $R$-band magnitude of -14 in the final 3.5 years before the explosion and $<0.56$ events over the final year. In contrast, precursors among SNe IIn have a $\gtrsim 5$ times higher rate. The kinetic energy required to unbind a low-mass stellar envelope is comparable to the radiated energy of a few-weeks-long precursor that would be detectable for the closest $\mathrm{SNe}$ in our sample. Therefore, mass ejections, if they are common in such $\mathrm{SNe}$, are radiatively inefficient or have durations longer than months. Indeed, when using 60-day bins, a faint precursor candidate is detected prior to SN 2012cs ( $\sim 2 \%$ false-alarm probability). We also report the detection of the progenitor of SN 2011dh that does not show detectable variability over the final two years before the explosion. The suggested progenitor of SN 2012P is still present, and hence is likely a compact star cluster or an unrelated object.
\end{abstract}

Key words: stars: mass-loss - supernovae: general - supernovae: individual (SN2011dh, SN2012P, SN2012cs, SN2013bb)

Supporting material: machine-readable tables

\section{INTRODUCTION}

Violent stellar activity, for example, precursor eruptions, during the years prior to the terminal explosion of a star as a supernova (SN) can give insight into the properties of progenitor stars at this crucial stage of their life. In case these eruptions are causally connected to the final explosion, they might even provide clues about the explosion mechanism. Indications for such luminous flares were reported previously, mainly among SNe IIn (e.g., Foley et al. 2007; Pastorello et al. 2007; Fraser et al. 2013; Mauerhan et al. 2013; Ofek et al. 2013, 2014), and a possible precursor was also reported for an SN Ic (Corsi et al. 2014). Here, we search for precursor eruptions, i.e., small explosions prior to the $\mathrm{SN}$, in the preexplosion light curves of $\mathrm{SNe} \mathrm{IIb}$.

The defining property of $\mathrm{SNe} \mathrm{IIb}$, the first known example of which was SN $1987 \mathrm{~K}$ (Filippenko 1988), is their spectral evolution. The spectrum first resembles that of a hydrogen-rich SN II and later turns into a helium-dominated spectrum similar to that of an SN Ib (e.g., Filippenko et al. 1993, 1994; Chornock et al. 2011; Ben-Ami et al. 2015; see Filippenko 1997 for a general review of SN spectra). This evidence suggests that the progenitors of SNe IIb likely consist of a helium core surrounded by a low-mass hydrogen envelope that expands, and consequently the hydrogen features fade away within a few weeks (e.g., Woosley et al. 1994; Tsvetkov et al. 2009; Chevalier \& Soderberg 2010; Bersten et al. 2012; Ergon et al. 2014a, 2014b; Marion et al. 2014; Nakar \& Piro 2014).

Another characteristic is that some SNe IIb show two optical photometric peaks in their light curve (e.g., SN 1993J, Wheeler et al. 1993; SN 2011dh, Arcavi et al. 2011; SN 2013df, Van Dyk et al. 2014). The first peak can be attributed to thermal emission from a shock-heated extended hydrogen envelope (Bersten et al. 2012; Nakar \& Piro 2014), while the second one is presumably powered by radioactivity. Nakar \& Piro (2014) suggest that the envelope's radius and mass can be inferred from the shape of the first peak. They have also shown that standard red giant or Wolf-Rayet progenitors cannot explain the observed light curves. Indeed, large photospheric radii of several hundred solar radii have been measured for several $\mathrm{SNe}$ IIb at early times (e.g., Woosley et al. 1994; Tsvetkov et al. 2009; Bersten et al. 2012; Horesh et al. 2013; Ergon et al. 2014a, 2014b). 
Additionally, an ultraviolet (UV) excess relative to the spectral shape in the optical has been observed for several $\mathrm{SNe}$ IIb whose UV spectra were obtained within the first month after explosion. It can be explained by the presence of a dense circumstellar medium (CSM) above the photosphere (Ben-Ami et al. 2015). Double-peaked light curves and excess UV radiation are, however, not observed for all $\mathrm{SNe} \mathrm{IIb}$ (I. Arcavi et al. 2015, in preparation; Ben-Ami et al. 2015). Moreover, Chevalier \& Soderberg (2010) argue that some SNe IIb show indications for much more compact progenitors having $R \approx 1 R_{\odot}$.

It is unclear how extended envelopes or dense CSM shells are created. They could consist either of material in hydrodynamic equilibrium (Bersten et al. 2012; Benvenuto et al. 2013) or of unbound material ejected during the interaction with a binary companion (e.g., Chevalier 2012), by a stellar wind, or by an instability related to the final stages of stellar burning (e.g., Rakavy \& Shaviv 1967; Arnett \& Meakin 2011a, 2011b; Quataert \& Shiode 2012; Shiode \& Quataert 2014). Such an instability can generate a precursor event that can release some of its energy in visible light.

A systematic search for precursor eruptions among SNe IIn has previously been performed by Ofek et al. (2014), with 6 likely precursor explosions observed in a sample of 16 objects. A quantitative analysis showed that, assuming a uniform population, at the $99 \%$ confidence level at least $98 \%$ of the $\mathrm{SNe}$ IIn exhibit one or several precursor events brighter than an absolute magnitude of -14 within the last 2.5 years prior to their explosion. These results apply to all SNe IIn, under the assumption that they form a homogeneous population that is well represented by the used sample. Furthermore, Ofek et al. (2014) find possible correlations between the amount of energy radiated in a precursor event and the SN rise time, peak magnitude, and radiated energy. If real, the correlation is consistent with the idea that SNe IIn are powered mainly by the interaction of the SN ejecta with a massive CSM created in precursor eruptions.

Only SNe having strong and persistent narrow hydrogen lines were included in the sample of Ofek et al. (2014). It was found recently that some core-collapse $\mathrm{SNe}$, dubbed "flashspectroscopy SNe," exhibit narrow hydrogen lines within the first days after the explosion that subsequently vanish (GalYam et al. 2014; D. Khazov et al. 2015, in preparation). This evolution might indicate that a dense CSM shell or an envelope is located only in the immediate vicinity of the progenitor star and is swept up by the ejecta shortly after the explosion. Owing to the short duration of the CSM interaction, these objects are not considered in the sample of SNe IIn of Ofek et al. (2014) and might not undergo precursor explosions as frequently. We note that the detection of flash-spectroscopy signatures provide further evidence for mass ejections prior to SN explosions.

In another search for precursors among SNe IIn by Bilinski et al. (2015), no pre-explosion events were found. However, with six objects their sample is smaller, and they do not provide an absolute-magnitude-dependent upper limit on the precursor rates, so a close comparison is not possible at this stage.

Here, we extend the search of Ofek et al. (2014) to SNe IIb. In Section 2, we explain how the SN sample was selected, and in Section 3, we describe the observations used in this analysis. Our search for precursor explosions is presented in Section 4. The pre-explosion observations of three nearby $\mathrm{SNe}$ are evaluated in more detail in Section 5. In Section 6, we calculate the sample control time and derive an upper limit on the precursor rate of SNe IIb. Section 7 discusses whether the ejection of a low-mass stellar envelope is likely detectable in this search, and Section 8 summarizes the results.

\section{SAMPLE SELECTION}

The sample is selected by initially considering all nearby SNe IIb detected either by the Palomar Transient Factory (PTF; Law et al. 2009; Rau et al. 2009) or announced in Astronomer's Telegrams or IAU Circulars since 2009 (i.e., after the start of the PTF project). SNe IIb are chosen based on their spectral evolution, and the main criterion is the appearance of helium features at 5876, 6678, and $7065 \AA$ a few weeks after the explosion. Tools such as the Supernova Identification Code (SNID; Blondin \& Tonry 2007) and Superfit (Howell et al. 2005) are used to compare our spectra to the spectra of known SNe IIb in case the spectral features are not obvious. We note that, in general, our SN classification is based on human decision, and hence may be biased. To increase the chances of detecting faint precursors, we restrict ourselves to nearby $\mathrm{SNe}$ with $z \leqslant 0.05$, corresponding to a luminosity distance of $220 \mathrm{Mpc}$. Only SNe having a large number of PTF observations prior to their explosion are selected. We require about 20 images (either before or long after the SN explosion) to construct a high-quality reference image, and another 20 science images before the explosion date.

Our final sample consists of $27 \mathrm{SNe}$ listed in Table 1. The majority were discovered by PTF, and additional SNe were found by the Lick Observatory Supernova Search ( $\mathrm{Li}$ et al. 2000; Filippenko et al. 2001), the Puckett Observatory Supernova Search, ${ }^{14}$ the La Silla Quest survey (LSQ; Baltay et al. 2013), the Catalina Real-time Transient Survey (Drake et al. 2009a), the Italian Supernova Search Project, ${ }^{15}$ and the Panoramic Survey Telescope and Rapid Response System (Pan-STARRS; Hodapp et al. 2004). For three SNe (SN 2011ef, SN 2012an, and LSQ 12fwb), no spectra are publicly available, and we rely on the classification published in Astronomer's Telegrams and Central Bureau Electronic Telegrams by Blanchard et al. (2011), Chen et al. (2012), and Hadjiyska et al. (2012). Representative spectra of all SNe discovered by PTF are shown in the Appendix (Yaron \& GalYam 2012). The spectra were acquired using various facilities that are listed in Table 5.

Our sample includes SN 2013cu (PTF 13ast), which has been classified as a flash-spectroscopy SN (Gal-Yam et al. 2014; D. Khazov et al. 2015, in preparation). After the initial CSM interaction, SN 2013cu evolves into an SN IIb (see the spectrum in the Appendix) and is thus included in this sample. Out of all core-collapse SNe having spectra within the first 10 days after their last nondetection, $\sim 14 \%$ show flash-spectroscopy signatures (D. Khazov et al. 2015, in preparation). It is therefore likely that other $\mathrm{SNe}$ in our sample for which no early spectra have been obtained belong to this group.

\section{OBSERVATIONS}

The observations used here were obtained with the 48 inch Oschin Schmidt telescope at Palomar Observatory (P48), as

\footnotetext{
14 http://www.cometwatch.com/supernovasearch.html

15 http://italiansupernovae.org/
} 
Table 1

Supernova Sample

\begin{tabular}{|c|c|c|c|c|c|c|c|c|c|c|c|}
\hline Name & $\begin{array}{l}\alpha(\mathrm{J} 2000) \\
(\mathrm{deg})\end{array}$ & $\begin{array}{c}\delta(\mathrm{J} 2000) \\
(\mathrm{deg})\end{array}$ & $z$ & $\begin{array}{c}\mathrm{DM} \\
(\mathrm{mag})\end{array}$ & $\begin{array}{c}E(B-V) \\
(\mathrm{mag})\end{array}$ & $\begin{array}{l}M_{R, \text { peak }} \\
\text { (mag) }\end{array}$ & $\begin{array}{c}t_{0} \\
\text { (day) }\end{array}$ & $\begin{array}{c}t_{\text {peak }} \\
\text { (day) }\end{array}$ & FAP & DP & Ref. Period \\
\hline PTF 09dxv & 347.144705 & +18.937131 & 0.0322 & 35.77 & 0.145 & $-18.0^{*}$ & 55079 & 55094 & 0.000 & & $>56041$ \\
\hline SN 2009nf, PTF 09gyp & 029.736494 & -07.282473 & 0.046 & 36.57 & 0.027 & -17.6 & 55138 & 55151 & 0.025 & & $>55835$ \\
\hline PTF 09hnq & 345.470095 & +14.413534 & 0.027 & 35.38 & 0.108 & $-17.5^{*}$ & 55157 & 55168 & 0.002 & & $>56449$ \\
\hline PTF 09ism & 176.149461 & +10.212143 & 0.03 & 35.61 & 0.075 & -17.4 & 55194 & 55200 & 0.000 & & $>56328$ \\
\hline PTF 10fqg & 190.457745 & +11.591142 & 0.0278 & 35.44 & 0.032 & -16.6 & 55302 & 55323 & 0.126 & & $>56712$ \\
\hline PTF 10qrl & 347.470125 & +13.132566 & 0.0396 & 36.23 & 0.076 & -17.0 & 55413 & 55427 & 0.027 & & $>56503$ \\
\hline PTF 10tzh & 257.305622 & +41.755139 & 0.034 & 35.89 & 0.025 & $-16.3^{*}$ & 55437 & 55459 & 0.002 & $\mathrm{y}$ & $>56360$ \\
\hline PTF 10xfl & 034.955874 & +15.295001 & 0.05 & 36.76 & 0.105 & $-18.2^{*}$ & 55456 & 55470 & 0.030 & & $>56533$ \\
\hline SN 2011dh, PTF 11eon & 202.521152 & +47.169782 & 0.001683 & 29.45 & 0.035 & -17.1 & 55713 & 55732 & 0.050 & $\mathrm{y}$ & $>56662$ \\
\hline SN 2011hg, PTF 11pdj & 347.953215 & +31.016708 & 0.0236 & 35.08 & 0.075 & & 55856 & & 0.000 & & $>56735$ \\
\hline PTF 11qju & 213.862526 & +36.408608 & 0.0282 & 35.48 & 0.089 & -17.5 & 55880 & 55902 & 0.007 & & $>56324$ \\
\hline SN 2012P, PTF 12os & 224.996177 & +01.890051 & 0.004533 & 32.04 & 0.051 & -16.3 & 55931 & 55952 & 0.006 & & $>56710$ \\
\hline PTF $12 f x j$ & 027.004256 & +35.708841 & 0.0148 & 34.06 & 0.056 & -17.4 & 55089 & 55102 & 0.054 & & $<55127$ \\
\hline SN 2012ey, PTF 12iqw & 036.210492 & +16.181395 & 0.027 & 35.38 & 0.179 & $-15.8^{*}$ & 56183 & 56198 & 0.000 & & $<55503$ \\
\hline PTF $12 \mathrm{jaa}$ & 338.364087 & +00.740223 & 0.0237 & 35.09 & 0.087 & $-15.6^{*}$ & 56190 & 56193 & 0.000 & $\mathrm{y}$ & $<55896$ \\
\hline PTF 13nu & 183.267741 & +32.613898 & 0.026 & 35.30 & 0.013 & -17.7 & 56356 & 56376 & 0.355 & & $<55896$ \\
\hline SN 2013bb, PTF 13aby & 213.058377 & +15.842080 & 0.01755 & 34.43 & 0.015 & -16.5 & 56369 & 56394 & 0.217 & & $>56766$ \\
\hline PTF 13ajn & 129.528692 & +66.526227 & 0.03 & 35.62 & 0.049 & $-17.3^{*}$ & 56385 & 56404 & 0.077 & $\mathrm{y}$ & $\begin{array}{c}<55183 \\
\&<56325\end{array}$ \\
\hline SN 2013cu, PTF 13ast & 218.495242 & +40.239672 & 0.0258 & 35.28 & 0.012 & -18.5 & 56414 & 56426 & 0.003 & & $<55042$ \\
\hline PTF 13ebs & 140.284454 & +49.592651 & 0.027 & 35.38 & 0.022 & $-16.9^{*}$ & 56606 & 56622 & 0.003 & & $>56780$ \\
\hline SN 2011ef & 352.737583 & +15.490083 & 0.0134 & 33.84 & 0.067 & $\cdots$ & 55760 & $\ldots$ & 0.095 & & $>56406$ \\
\hline SN 2012an & 261.042625 & +59.001916 & 0.0111 & 33.43 & 0.030 & $\cdots$ & 55978 & $\cdots$ & 0.000 & & $>56778$ \\
\hline SN 2012cs & 232.990208 & +68.245222 & 0.0218 & 33.56 & 0.027 & $\cdots$ & 56053 & $\ldots$ & 0.041 & & $<55052$ \\
\hline LSQ 12 fwb & 006.355083 & +06.707194 & 0.03 & 35.62 & 0.024 & $\ldots$ & 56233 & $\cdots$ & 0.114 & & $<55430$ \\
\hline LSQ $12 \mathrm{htu}$ & 152.904625 & -07.386555 & 0.04 & 36.26 & 0.034 & $\cdots$ & 56282 & $\ldots$ & 0.006 & & $>56712$ \\
\hline SN 2013df & 186.622208 & +31.227305 & 0.002388 & 31.23 & 0.019 & -16.8 & 56447 & 56470 & 0.965 & $\mathrm{y}$ & $<56388$ \\
\hline PS1-14od & 050.275958 & -07.282611 & 0.02 & 34.72 & 0.059 & $\ldots$ & 56713 & $\ldots$ & 0.000 & & $<55089$ \\
\hline
\end{tabular}

Notes. The SN sample. $\alpha(\mathrm{J} 2000)$ and $\delta(\mathrm{J} 2000)$ are the J2000.0 R.A. and decl., respectively; $z$ is the SN redshift obtained from spectroscopy. DM, the distance modulus, is derived from the redshift with $H_{0}=69.33 \mathrm{~km} \mathrm{~s}^{-1} \mathrm{Mpc}^{-1}, \Omega_{M}=0.24$, and $\Omega_{\Lambda}=0.71$ (Hinshaw et al. 2013). The only exceptions are the three closest $\mathrm{SNe}$ and SN 2012cs, where redshift-independent distance measurements of the host galaxies are available on NED. $E(B-V)$ is the Galactic extinction taken from Schlegel et al. (1998). $M_{R}$, peak is the absolute $R$-band magnitude of the brightest detection; asterisks indicate that the peak is not well observed and the SN might be considerably brighter. $t_{0}$ is the MJD of the approximate explosion date estimated by picking a date between the last nondetection and the first detection; thus, for some SNe the uncertainty in $t_{0}$ can be many days. $t_{\text {peak }}$ is the MJD of the brightest detection, where only the second peak is considered in the case of a double-peaked light curve. FAP, the false-alarm probability, is the probability of detecting a false precursor candidate by coadding images in 15-day bins as estimated using the bootstrap method (see Section 4.1). In the penultimate column SNe for two peaks observed in the light curve are marked with a "y", and the last column specifies the chosen reference period. Below the solid line we list SNe added to our sample from the literature.

References. PTF 09hnq, PTF 10fqg, PTF 10qrl, PTF 10tzh, PTF 10xfl, PTF 11qju, PTF 12jaa, PTF 13nu, PTF 13ajn, and PTF 13ebs: reported here for the first time. PTF 09dxv: Arcavi et al. (2010).

SN 2009nf (PTF 09gyp): Drake et al. (2009b); Arcavi et al. (2010).

PTF 09ism: Arcavi et al. (2010).

SN 2011dh (PTF 11eon): Arcavi et al. (2011); Griga et al. (2011).

SN 2011hg (PTF 11pdj): Ciabattari \& Mazzoni (2011); Gal-Yam et al. (2011); Marion \& Berlind (2011); Tomasella et al. (2011).

SN 2012P (PTF 12os): Arcavi et al. (2012); Borsato \& Nascimbeni (2012); Dimai et al. (2012).

SN 2013bb (PTF 13aby): Howerton et al. (2013); Elias-Rosa et al. (2013).

SN 2011ef: Blanchard et al. (2011); Parrent et al. (2011).

SN 2012an: Chen et al. (2012); Jha et al. (2012); Newton et al. (2012).

SN 2012cs: Rich et al. (2012).

SN 2012ey: Howerton et al. (2012); Turatto et al. (2012).

LSQ 12fwb: Hadjiyska et al. (2012).

LSQ 12htu: Le Guillou et al. (2012).

SN 2013cu: Gal-Yam et al. (2014).

SN 2013df: Ciabattari et al. (2013); Van Dyk et al. (2013); Van Dyk et al. (2014); Morales-Garoffolo et al. (2014); Ben-Ami et al. (2015).

PS1-14od: Campbell et al. (2014).

part of the PTF project. PTF searches for transient sources by visiting selected fields of the sky regularly over the duration of several months. The data-reduction procedure is described by Laher et al. (2014) and the photometric calibration by Ofek et al. (2012a, 2012b). We only use observations in the $R$ band and neglect $<15 \%$ of the data in the $g$ band to simplify this analysis. For every SN, a reference period containing at least 20 observations is chosen. If possible, we use data well after the SN has faded to construct the reference image, but when no such data are available, we instead resort to the oldest 
Table 2

PTF Observations

\begin{tabular}{|c|c|c|c|c|c|c|c|}
\hline Name & $\begin{array}{c}\text { MJD- } t_{0} \\
\text { (day) }\end{array}$ & $\begin{array}{l}\text { MJD } \\
\text { (day) }\end{array}$ & $\begin{array}{c}m_{\mathrm{PTF}, R} \\
(\mathrm{mag})\end{array}$ & $\begin{array}{c}m_{\mathrm{PTF}, R} \text { Err } \\
(\mathrm{mag})\end{array}$ & $\begin{array}{l}\text { Lim Mag } \\
\text { (mag) }\end{array}$ & $\begin{array}{c}\text { Flux } \\
\text { (counts) }\end{array}$ & $\begin{array}{l}\text { Flux Err } \\
\text { (counts) }\end{array}$ \\
\hline PTF 09dxv & -47.876 & 55031.304 & 25.34 & 31.38 & 21.09 & 2.7 & 76.9 \\
\hline PTF 09dxv & -45.711 & 55033.469 & 78.65 & $\ldots$ & 21.38 & -9.0 & 59.2 \\
\hline PTF 09dxv & -45.705 & 55033.475 & 21.69 & 0.49 & 21.37 & 133.2 & 59.7 \\
\hline PTF 09dxv & -43.762 & 55035.418 & 79.45 & $\ldots$ & 21.46 & -18.8 & 54.9 \\
\hline
\end{tabular}

Note. Flux residuals in the pre-explosion light curves of all SNe in our sample. Magnitudes are calculated as "asinh magnitudes" (Lupton et al. 1999), and have meaning only when smaller than the limiting magnitude. The limiting magnitude here is at the $3 \sigma$ level.

(This table is available in its entirety in machine-readable form.)

pre-explosion images. For PTF 13ajn, a considerable number of observations were acquired with two CCDs, and we hence define two different reference periods. All reference periods are listed in the last column of Table 1.

For each SN, the reference image is created by coadding observations within the reference period. This image is subtracted from all science images and the (positive or negative) flux residuals at the SN location are measured using forced point-spread function (PSF) photometry. A correction for Galactic extinction according to Cardelli et al. (1989) and Schlegel et al. (1998) is applied to all fluxes and magnitudes in this paper.

The observations and their flux residuals are listed in Table 2. They were obtained up to 3.5 years prior to the SN explosion. Figures 1-3 display the pre-explosion light curves in 15-day bins. Filled circles correspond to bins containing six or more observations, while open circles represent bins with fewer data points. Crosses around zero mark the estimated $5 \sigma$ noise level, whose calculation is described in Section 4.1.

\section{PRECURSOR SEARCHES}

The precursor search follows to a large extent the methods of Ofek et al. (2014). In a first search, we look for precursor events in individual images. In a second search channel, observations are combined once in 15-day and once in 60-day bins to gain sensitivity to faint precursor events. An advantage of the binned search is that it allows us to estimate the noise level from the data itself, which is more precise than the formal errors calculated by the image-subtraction pipeline. The only drawback of binning the data is that a minimal precursor duration has to be assumed and we lose sensitivity to shorter eruptions.

Both search methods are presented in Section 4.1 and the results are described in Section 4.2. Additional tests and cross checks on the data are explained in Section 4.3. This search uses tools available in the MATLAB astronomy and astrophysics package (Ofek 2014).

\subsection{Search Methods}

In the unbinned search, we have to rely on the statistical errors estimated by the image-subtraction pipeline. These uncertainties are based on Poisson noise, and several additional uncertainties are neglected. Those neglected error sources include errors on the fitted PSF and correlations between neighboring pixels induced by smoothing images before the subtraction with a kernel takes place (e.g., Alard \& Lupton 1998; Bramich 2008). Therefore, the calculated errors often underestimate the fluctuations in the data, and here we scale them up to obtain a more realistic error estimate.

We calculate the standard deviation of the complete preexplosion light curve using the errors from the imagesubtraction pipeline and compare it to the actual scatter in the data estimated using the bootstrap method (Efron 1982). When applying the bootstrap method, we resample the pre-explosion light curve 1000 times by randomly assigning flux residuals to the observation times. Flux residuals can be drawn several times. We then calculate the average flux of every simulated light curve. The average fluxes are distributed according to a normal distribution whose standard deviation is the bootstrap error on the average flux. When using the bootstrap technique, we implicitly assume that the pre-explosion light curves are dominated by statistical fluctuations and that eventual precursors have a negligible influence on the light curves' standard deviation. We find that the bootstrap error is always larger than the standard deviation calculated from the Poisson errors. For many $\mathrm{SNe}$, the difference is approximately a factor of two. The errors on the individual flux residuals are scaled up accordingly, and we require a $5 \sigma$ deviation above zero for the detection of a precursor.

We caution that these flux residuals (even in the absence of a precursor) do not follow a Gaussian distribution. Outliers can be caused in a number of ways, such as by atmospheric conditions, cosmic rays hitting the detector, or imperfect image subtractions. Large deviations are rare, however, and they can be identified by inspecting the image or verifying that a precursor candidate is detected in several subsequent images.

In the second search channel, the sensitivity of the search is increased by coadding flux residuals in time bins. Our coaddition method preserves more information relative to simple image coaddition and has common ground with the optimal coaddition method described by B. Zackay \& E. O. Ofek (2015, in preparation). Following Ofek et al. (2014), we choose a bin size of 15 days, which means that precursor events having shorter durations might be missed even if their luminosity is above the quoted sensitivity. In addition, we repeat the analysis using 60-day bins to search for long-lasting faint precursors.

For the binned searches, bootstrap errors are calculated for individual bins, which is more reliable than scaling up the Poisson errors estimated by the image-subtraction pipeline. To get a sound error estimate with the bootstrap method, a bin 

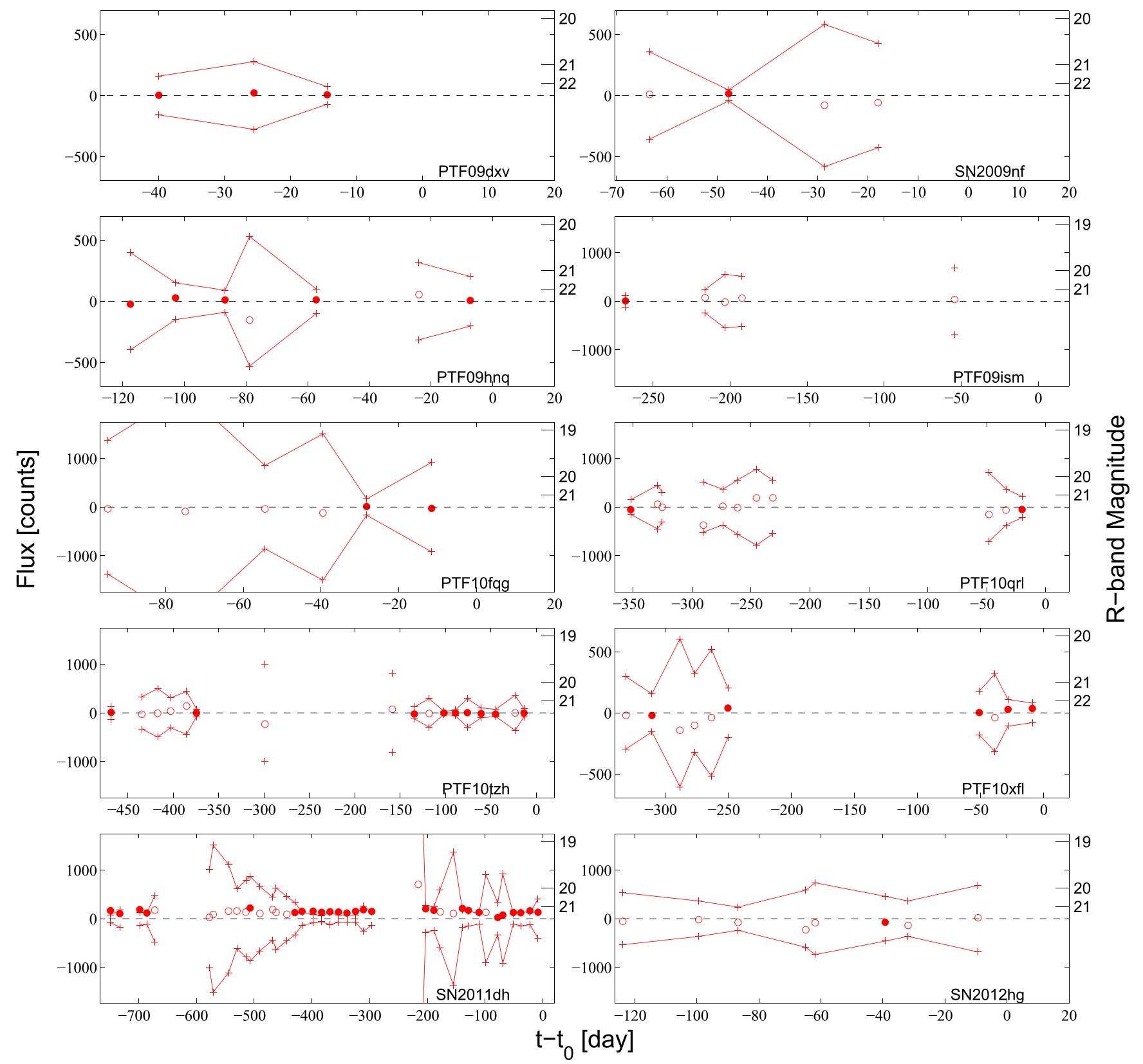

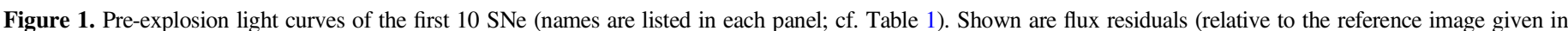

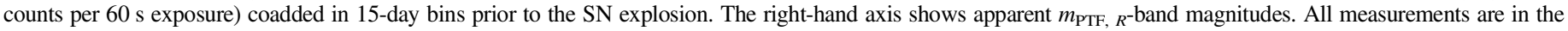

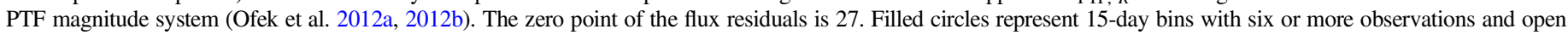

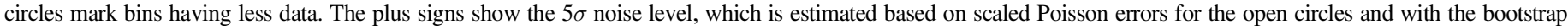

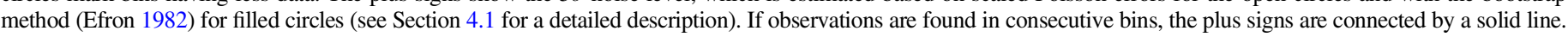
A few very large error regions are outside the plotted range to improve readability. Figures 2 and 3 show the pre-explosion light curves of the remaining SNe.

must contain several entries. Therefore, in the following, only bins with at least six observations are considered.

As before, we require a $5 \sigma$ deviation for the detection of a precursor, with the difference being that the noise level for every bin is estimated with the bootstrap method. To calculate a false-alarm probability, we randomly combine observation times and flux residuals from the entire pre-explosion light curve 1000 times, bin them in 15-day bins, and look for precursors in these scrambled light curves. The probabilities for detecting one or several false precursor candidates per SN are listed in Table 1 . This test, however, only leads to a valid result if the pre-explosion light curve is dominated by statistical fluctuations and its mean flux is consistent with zero.

The binned pre-explosion light curves are shown in Figures 1-3. In addition, bins containing fewer than six observations are marked by open circles. For these bins, the standard deviation of the mean flux is calculated based on the scaled Poisson errors.

\subsection{Precursor Candidates}

When applying the precursor searches described in Section 4.1 to the $\mathrm{SNe}$ in our sample, no precursor candidates are 

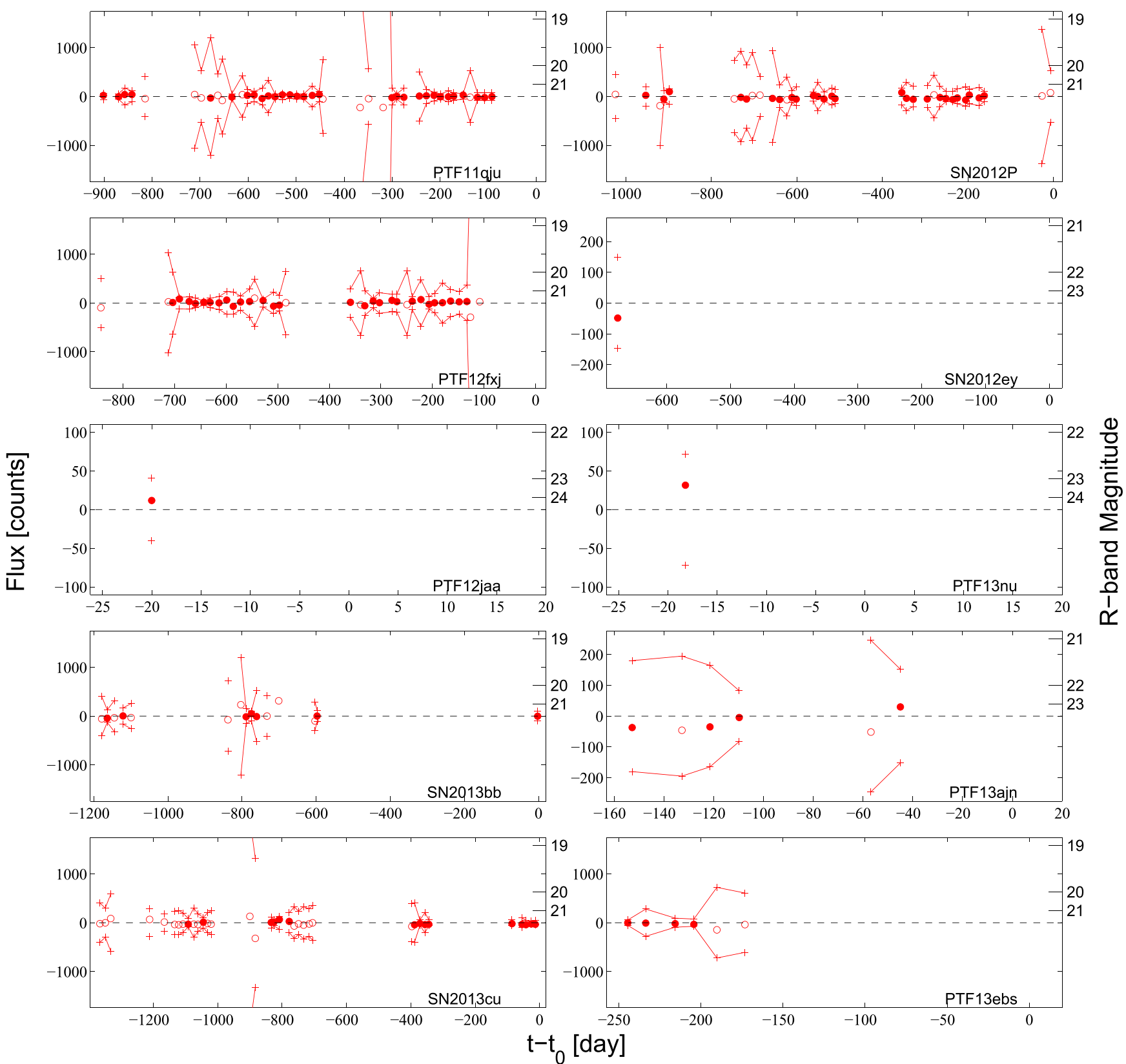

Figure 2. Figure 1, continued.

found in the unbinned search or when using 15-day bins; however, one precursor candidate is discovered when binning observations in 60-day bins.

In the unbinned pre-explosion light curves (not shown; flux residuals given in Table 2), no single observation has a flux above the $5 \sigma$ noise level estimated based on the scaled Poisson errors. From the nondetection of events above this threshold, we can infer an upper limit on the false-alarm rate for this search channel. Using the Poisson single-sided upper limits (Gehrels 1986), the expected number of precursors is smaller than 2.3 at the $90 \%$ confidence level when no event is observed. Since we evaluate in total 3152 pre-explosion observations, the false-alarm probability is smaller than 2.3 / $3152 \approx 0.0007$ per observation.

We find that even when lowering the threshold of this search to $3 \sigma$, only one single flux residual reaches the threshold. The most significant subthreshold event is a $4.2 \sigma$ deviation in the light curve of SN 2012P, 654 days before the SN explosion. A second observation with similar limiting magnitude only two hours earlier, however, does not yield a detection. The two following observations three days later are just as deep, but neither show signs of a flare. Given the number of trials, this event is most likely spurious. We nevertheless conservatively maintain the $5 \sigma$ threshold when calculating limits on the precursor rate in Section 6. Potential precursor events below the quoted flux threshold of our search do not affect the validity of the calculated limits.

We also do not find precursor candidates above the $5 \sigma$ noise level when using 15-day bins. The only exception is the preexplosion light curve of SN 2011dh, the nearest object in our sample. Its progenitor star is detected in coadded images, and in 17 of the bins the noise level is low enough to allow a significant detection. The average flux of the pre-explosion light curve of SN 2011dh is $17 \sigma$ above zero and the progenitor 


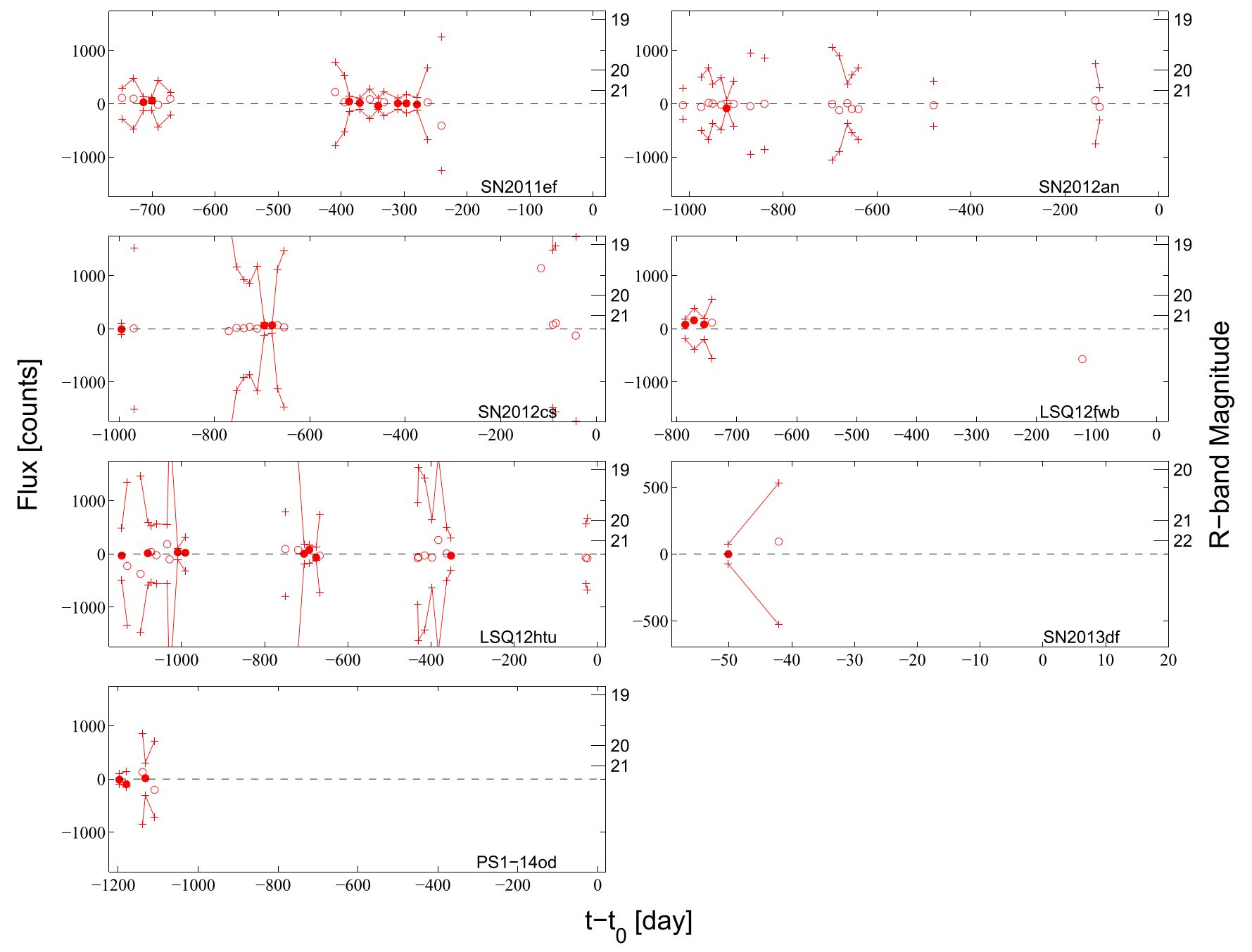

Figure 3. Figures 1 and 2, continued. Here we show the PTF pre-explosion light curves of SNe IIb found in the literature.

is hence clearly detected. In Section 5.1, we account for the progenitor's average flux, and all precursor candidates vanish, as can be seen in Figure 4.

When increasing the bin size to 60 days, one precursor candidate is detected marginally above the $5 \sigma$ threshold in the pre-explosion light curve of SN 2012cs. Figure 5 shows the binned light curve as well as the unbinned observations. For 60-day bins, the noise level is low enough to show a flux excess approximately 680 days before the SN explosion. The 16 observations in this bin are nearly all at the same flux level. The excess is, hence, not caused by individual observations, but many points contribute to it as it is expected from a longlasting precursor event. The median flux in this bin is 62 counts, which corresponds to a magnitude of $m_{\mathrm{PTF}, R}=22.51$. SN 2012cs has a redshift of 0.0218 . The absolute magnitude of the possible precursor is $M_{\mathrm{PTF}, R}=-11.0$, and its luminosity is $L_{\text {prec. }}=7 \times 10^{39} \mathrm{erg} \mathrm{s}^{-1}$ (no bolometric correction applied). A duration of about 60 days seems consistent with the unbinned light curve, and the radiated energy amounts to $4 \times 10^{46} \mathrm{erg}$ during this period. If real, the event is hence slightly fainter than precursors detected so far (compare to, e.g., Ofek et al. 2014), and its radiated energy is similar to the least energetic detected precursors.

Since the event is barely above the significance threshold of this search, it might be a false detection. The false-alarm probability estimated for SN 2012cs and a bin size of 60 days is $1.8 \%$. Its calculation is, however, based on the assumption that the pre-explosion light curve is dominated by statistical fluctuations. If the event is real, a considerable fraction of the observations (16 out of 52) are systematically shifted upward. A real precursor can thus enhance the false-alarm probability. We conclude that the detected candidate is consistent with an astrophysical precursor event. However, due to the marginal detection, we cannot decide whether or not it is real. No further precursor candidates are found. We focus in the following on the nondetection of precursors using 15-day bins, which allows us to constrain the rate of bright precursors. The detected possible precursor is below the sensitivity threshold of this analysis and hence does not affect the limits. The pre-explosion light curves of all $27 \mathrm{SNe} \mathrm{IIb}$ are shown in Figures 1-3, and the upper edge of the error band corresponds to the sensitivity of the search with a bin size of 15 days.

\subsection{Additional Tests}

In addition to the tests described in Section 4.1, we check whether the pre-explosion light curves are consistent with an average flux of zero, and we look for autocorrelations in the unbinned light curves. Both tests, described below, do not 


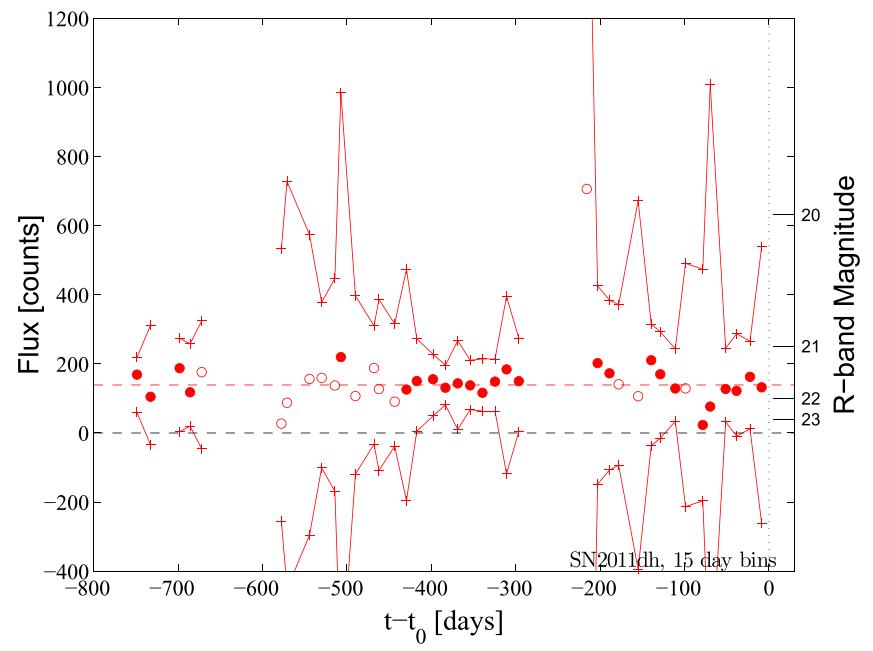

Figure 4. Binned pre-explosion light curve of SN 2011dh. The reference images are chosen to be after the explosion; hence, the flux of the progenitor has not been subtracted. No observations deviate by more than $5 \sigma$ from the mean flux.

reveal hints for detections or raise concerns about the data quality.

Initially, the complete pre-explosion light curves of several SNe were inconsistent with zero when calculating its average flux and its bootstrap error. In some cases, the SN was still present in the chosen reference images, and we instead resorted to a pre-explosion reference. For PTF 10qrl, we use preexplosion images since the SN location is at the very edge of the $\mathrm{CCD}$ in the post-explosion images, affecting the image subtraction. Also, the average pre-explosion fluxes of several sources show deviations marginally below the $3 \sigma$ threshold. This may affect the false-alarm probabilities of the binned search (shown in Table 1). We verify, however, that no additional precursor candidates are found when subtracting the mean pre-explosion flux from all of the residuals.

Furthermore, we look for autocorrelations in the unbinned pre-explosion light curves, where we calculate the correlation between an observation and the five following observations. The only deviation above the $3 \sigma$ threshold is found for PTF 10qrl with a lag of two observations. This might, however, be caused by chance. The autocorrelation is calculated for all 27 SNe with five different lags each, which means that at the $3 \sigma$ level 0.36 false detections are expected. The probability to detect one or several events above the $3 \sigma$ threshold is therefore $30 \%$. Moreover, when looking for autocorrelations in time bins of three days instead of indexing the observations, the correlation vanishes. PTF 10qrl is located at a redshift of 0.05 and is hence is too far away for the detection of the progenitor star.

We conclude that no indications for precursor detections are found in these additional tests.

\section{PROGENITOR DETECTIONS AND VARIABILITY}

For the three nearest $\mathrm{SNe}$ in our sample, here, we explore the possibility of detecting the progenitor star and calculating limits on its variability.

\subsection{SN $2011 d h$}

SN 2011dh was discovered on 2011 May 31 in the nearby galaxy M51 at a distance of $7.8 \mathrm{Mpc}$, less than $15 \mathrm{hr}$ after a

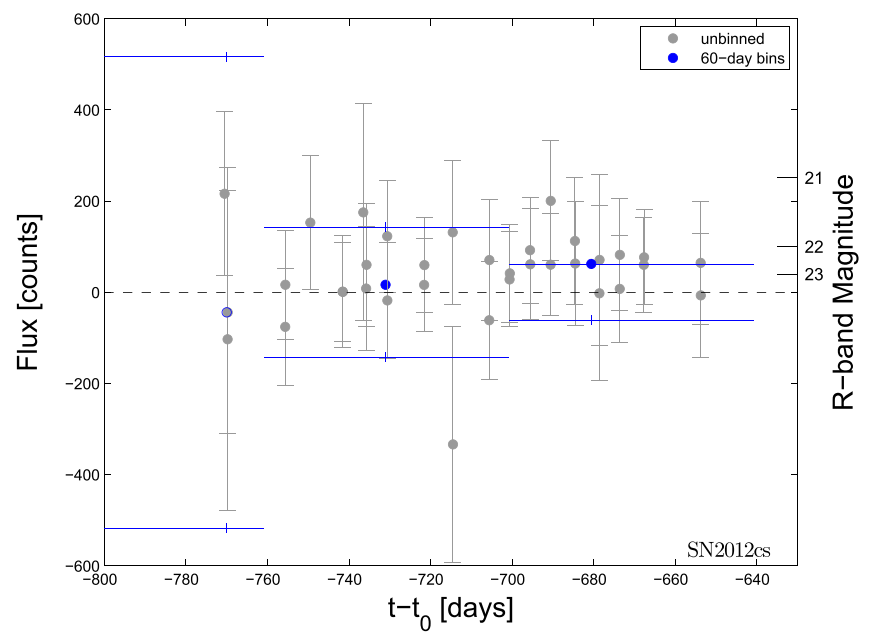

Figure 5. Section of the pre-explosion light curve of SN 2012cs. The gray error bars show the scaled-up $1 \sigma$ Poisson errors on the individual observations. The blue lines indicate the bin position as well as the $5 \sigma$ noise level for the binned light curve. Open dots represent bins containing fewer than six observations for which the noise level is estimated based on the scaled Poisson errors. Filled dots correspond to bins with at least six observations, and the bootstrap method was used to estimate the noise level. A detection, marginally above the $5 \sigma$ threshold, is obtained around day -680 . The false-alarm probability of SN 2012 cs is $1.8 \%$ when using 60-day bins.

nondetection down to a limiting magnitude of $m_{\mathrm{PTF}, g}=21.4$ (Arcavi et al. 2011). The progenitor star is clearly detected both by the Hubble Space Telescope (HST; Li et al. 2011; Maund et al. 2011; Van Dyk et al. 2011) and in ground-based observations (e.g., the Large Binocular Telescope, Szczygieł et al. 2012; the Northern Optical Telescope, Ergon et al. 2014a; and PTF). The progenitor is a yellow supergiant (Van Dyk et al. 2013). Moreover, a flux excess has been measured in the fading SN light curve in blue bands and has been attributed to the presence of a binary companion star (Folatelli et al. 2014). The companion presumably has $M_{\mathrm{F} 225 \mathrm{~W}}=-5.11 \pm 0.29 \mathrm{mag}$ and $M_{\mathrm{F} 336 \mathrm{~W}}=-4.66 \pm 0.29 \mathrm{mag}$, consistent with a B star having $10 M_{\odot}<M<16 M_{\odot}$ (Folatelli et al. 2014).

There are 373 PTF $R$-band observations that span the last two years before the explosion of SN 2011dh. Our reference is based on observations obtained starting 949 days after the explosion, when the SN has faded away. The progenitor star is hence not present in the reference image, so its flux is not subtracted from the pre-explosion light curve. All flux residuals are listed in Table 2.

The progenitor is not detected in individual images; however, in the binned light curve shown in Figure 4 the $\mathrm{SN}$ is significantly detected in many bins. The average unabsorbed flux of the progenitor is $139 \pm 10$ counts, corresponding to $m_{\mathrm{PTF}, R}=21.82 \pm 0.08 \mathrm{mag}$, where the uncertainty is the $1 \sigma$ bootstrap error. During the HST observations in 2005, the progenitor star was detected in the F658N band with a magnitude of $m_{\mathrm{F} 658 \mathrm{~N}}=21.39 \pm 0.02$ (Van Dyk et al. 2011), corresponding to a flux of $(4.94 \pm 0.09) \times 10^{-18} \mathrm{erg} \mathrm{s}^{-1} \mathrm{~cm}^{-2} \AA^{-1}$ at an effective wavelength of $6579.5 \AA$ (flux taken from Benvenuto et al. 2013). This value is comparable to our measurement, translating into a flux of $(5.54 \pm 0.35) \times 10^{-18} \mathrm{erg} \mathrm{s}^{-1} \mathrm{~cm}^{-2} \AA^{-1}$ at the same effective wavelength.

To search for precursors on top of the progenitor flux, the $5 \sigma$ error band is centered around the star's average flux. No single or binned observations exceed this error band, and hence no 
precursors are found. One bin around day 215 before the explosion has an average flux of more than 600 counts. It, however, only contains two flux residuals that both have large errors. We hence conclude that this point is caused by two lowquality observations. Its deviation from zero is less than $2 \sigma$.

Moreover, we search for periodic variability by calculating the power spectrum of the pre-explosion flux residuals. No significant variability is found at any timescale either in the binned or unbinned data.

Szczygieł et al. (2012) analyze pre-explosion observations taken with the Large Binocular Telescope within three years before the SN explosion. With data at five different epochs, they suggest a continuous fading at a rate of $0.039 \pm$ $0.006 \mathrm{mag} \mathrm{yr}^{-1}$ in the $R$ band. However, in a sample of stars of comparable luminosity, large or larger dimmings are observed for $5 \%$ of the stars, and about $16 \%$ of the stars have a photometric rms value larger than that of the progenitor of SN 2011dh (Szczygieł et al. 2012). Therefore, it is possible that this variability is not real. A fading in the last century before the SN explosion is expected as the envelope slowly responds to the rapid changes in the stellar core during the last stages of nuclear burning. The decline rates predicted by simulations are, however, typically 100 times smaller than the measured dimming rate (Szczygieł et al. 2012).

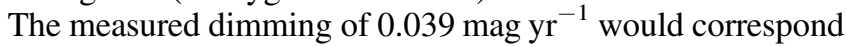
to a change of only nine counts between our first and last observation, comparable to the uncertainty in the average flux. To check whether our observations do favor a dimming, the binned pre-explosion light curve is fitted both with a constant flux and with a dimming at the measured rate. The results are very similar, with $\chi^{2} /$ dof $=35.4 / 39$ for the constant model compared to $\chi^{2} /$ dof $=34.4 / 39$ for the fading light curve. With $\Delta \chi^{2} \approx 1$, we cannot confirm or eliminate the fading hypothesis of Szczygieł et al. (2012).

\section{2. $S N 2013 d f$}

The second-nearest object in our sample is SN 2013df at a distance of $18.2 \mathrm{Mpc}$. No attempt can be made to detect the progenitor since we do not have potential reference images after the $\mathrm{SN}$ has faded. The progenitor, a $500 R_{\odot}$ cool supergiant, was identified by Van Dyk et al. (2014) in preexplosion HST images with an absolute magnitude of $M_{\mathrm{HST}, V} \approx-6.8$.

\subsection{SN $2012 P$}

At the position of SN 2012P, a potential progenitor star was detected in archival HST observations from 2005 March 10 (Van Dyk et al. 2012). The source has an absolute magnitude of $M_{\mathrm{HST}, V} \approx-9.1$, and its luminosity and colors are consistent with a very luminous supergiant star or a star cluster.

Interpolating the $V$ - and $I$-band magnitudes reported by Van Dyk et al. (2012), a PTF $R$-band magnitude of $m_{\mathrm{PTF}, R}=22.74$ is expected, corresponding to a signal of 50 counts per observation. The weighted average flux of the 308 preexplosion observations is, however, $-17 \pm 10$ counts per observation ( $\sigma \sigma$ bootstrap error). Hence, we conclude that the source reported by Van Dyk et al. (2012) is still present in the reference image, which consists of observations more than 779 days after the $\mathrm{SN}$ explosion. The source close to the SN position could be either a compact star cluster as mentioned by
Van Dyk et al. (2012), a luminous binary companion, or an unrelated object.

Based on the PTF pre-explosion observations in the last three years before the explosion of SN 2012P, we can exclude precursor explosions with an absolute $R$-band magnitude of $M_{\mathrm{PTF}, R} \leqslant-11 \quad\left(L \geqslant 8 \times 10^{39} \mathrm{erg}^{-1}\right.$ without a bolometric correction) during $30 \%$ of the time.

\section{CONTROL TIME AND PRECURSOR RATES}

Following Ofek et al. (2014), we combine the results of the individual $\mathrm{SNe}$ and calculate an upper limit on the precursor rate of $\mathrm{SNe} \mathrm{IIb}$. Hereby, we implicitly assume that the progenitors of $\mathrm{SNe} \mathrm{IIb}$ form a uniform population and that our SN sample provides a good representation of this population. Both presumptions are likely not fulfilled and the resulting rate estimate should hence be regarded with caution.

To address the inhomogeneity of the progenitor population, we additionally calculate an upper limit only for SNe IIb with observed double-peaked light curves since this is a strong indication for an extended envelope (Nakar \& Piro 2014).

Moreover, faint SNe IIb are likely underrepresented in this sample; however, our sample does cover a wide range of peak luminosities and light-curve shapes. This analysis is thus not restricted to any certain subgroup of SNe IIb. The advantage of a rate estimate is that we potentially gain information on the $\mathrm{SNe} \mathrm{IIb}$ as a class and we are able to quantify the results of this analysis.

To calculate a precursor rate for all $\mathrm{SNe} \mathrm{IIb}$, we estimate the control time-the time during which a precursor above a certain absolute magnitude can be detected. The following calculations are described for a bin size of 15 days. When using 60-day bins, all calculations and results are similar except for the detection of the faint precursor candidate. All resulting upper limits or precursor rates are summarized in Table 3 for both bin sizes.

We adopt a minimal precursor duration of 15 days and bin the observations accordingly. For bins with six or more observations, the $1 \sigma$ noise level is calculated using the bootstrap method and is then multiplied by a factor of five to estimate a $5 \sigma$ level. For bins with fewer observations, we determine the standard deviation based on the scaled Poisson errors estimated by the image-subtraction pipeline (see Section 4.1). The upper edge of the noise level corresponds to the $5 \sigma$ limiting magnitude per bin. The control time is formally given as

$$
\begin{aligned}
t_{\text {search }}\left(M_{R}\right) & =\sum_{i=1}^{N_{\text {bin }}, N_{\mathrm{SN}}} t_{i, \text { search }}\left(M_{i, R}\right) \\
\text { with } t_{i, \text { search }}\left(M_{i, R}\right) & = \begin{cases}15 \text { days, } & \text { if } M_{i, R}<M_{R} \\
0 \text { days, } & \text { otherwise, }\end{cases}
\end{aligned}
$$

where $M_{R}$ is the absolute magnitude at which the control time is calculated and $M_{i, R}$ is the $5 \sigma$ limiting magnitude in bin $i$. The sum runs over all $N_{\text {bin }}$ light-curve bins per $\mathrm{SN}$ and all $N_{\mathrm{SN}} \mathrm{SNe}$ in this sample. The mean observation time of the bin relative to the explosion date, the limiting magnitude, and the number of observations per bin are listed in Table 4 (unbinned observations are given in Table 2).

From the search results described in Section 4.2 and the light curves shown in Figures 1-3, we know that during the control time no precursors were detected above the estimated noise 
Table 3

$N_{\text {prec }}$, Average Number of Precursors per SN

\begin{tabular}{|c|c|c|c|c|c|}
\hline \multirow{2}{*}{$\begin{array}{l}\text { Time Interval } \\
\text { (year) }\end{array}$} & \multicolumn{2}{|c|}{ SNe IIb, Bright Precursors (-14 mag) } & \multicolumn{2}{|c|}{ SNe IIb, Faint Precursors $(-10 \mathrm{mag})$} & \multirow{2}{*}{$\begin{array}{c}\text { SNe IIn }(-14 \text { mag } \\
\text { 15-day bins }\end{array}$} \\
\hline & 15-day bins & 60-day bins & 15-day bins & 60-day bins & \\
\hline-3.5 to 0 & $<0.86$ & $<0.51$ & $<5.03$ & $1.52_{-1.26,-1.49}^{+3.50,+7.12}$ & ... \\
\hline-1 to 0 & $<0.56$ & $<0.36$ & $<2.95$ & $<2.00$ & $2.8_{-1.3,-2.1}^{+2.2,+4.4}$ \\
\hline$-1 / 3$ to 0 & $<0.52$ & $<0.29$ & $<2.08$ & $<2.33$ & $2.5_{-1.2,-1.8}^{+2.0,+4.0}$ \\
\hline-2 to -1 & $<0.70$ & $<0.47$ & $<3.12$ & $1.01_{-0.84,-0.99}^{+2.33,+4.75}$ & $\ldots$ \\
\hline
\end{tabular}

Notes. Upper limit on the allowed number of precursors $N_{\text {prec }}$ within the respective time interval (rates from Figure 7 (a) multiplied by time interval) compared to the measured number of precursors prior to SNe IIn (Ofek et al. 2014). The limits apply to precursors with a minimal duration of 15 and 60 days, respectively. The constraints on long precursors are stronger since more observations are coadded, which leads to a better sensitivity. All limits are at the $90 \%$ confidence level, and the quoted uncertainties correspond to the $1 \sigma$ and $2 \sigma$ Poisson errors (Gehrels 1986).

Table 4

Precursor Search Control Time

\begin{tabular}{lcccc}
\hline \hline Name & $\begin{array}{c}\Delta t \\
(\mathrm{day})\end{array}$ & $\begin{array}{c}m_{\mathrm{PTF}, R} \\
(\mathrm{mag})\end{array}$ & $\begin{array}{c}\text { Abs. } \\
M_{\mathrm{PTF}, R} \\
(\mathrm{mag})\end{array}$ & $N_{\text {meas }}$ \\
\hline PTF 09dxv & -40.0 & 21.49 & -14.28 & 20 \\
PTF 09dxv & -25.5 & 20.90 & -14.87 & 23 \\
PTF 09dxv & -14.4 & 22.38 & -13.39 & 14 \\
SN 2009nf & -63.4 & 20.61 & -15.96 & 4 \\
SN 2009nf & -47.7 & 22.87 & -13.70 & 8 \\
SN 2009nf & -28.6 & 20.08 & -16.49 & 3 \\
SN 2009nf & -18.0 & 20.41 & -16.16 & 3 \\
PTF 09hnn & -117.5 & 20.50 & -14.88 & 9 \\
\hline
\end{tabular}

Notes. Listed are the SN name, the mean observation time of the 15-day bin relative to the explosion date, the apparent and absolute magnitude down to which precursors can be excluded, and the number of observations per bin. The limiting magnitudes are at the $5 \sigma$ level estimated from Poisson errors for bins with less than six observations and with the bootstrap method otherwise (Section 4.1). They correspond to the upper + signs in Figures 1-3, and their cumulative distribution is shown in Figure 6.

(This table is available in its entirety in machine-readable form.)

level when using 15-day bins. The control time $t_{\text {search }}$ for the complete sample as a function of the precursor magnitude is depicted in Figure 6, where $t_{\text {search }}$ is defined as the bin duration of 15 days multiplied by the number of bins in which a precursor of the respective magnitude would have been detected. For example, at an absolute magnitude of -14 and considering the full time range of 3.5 years prior to the $\mathrm{SN}$ explosion (thick black line in Figure 6), a control time of 9.2 years has been accumulated; in our sample, there are two hundred twenty-three 15-day bins in which precursors having an absolute magnitude of -14 or less can be excluded.

According to the amount of data and the SN distance, the objects in our sample contribute differently to the magnitudedependent control time. The five $\mathrm{SNe}$ with the longest control time (PTF 11qju, SN 2011dh, PTF 12fxj, SN 2012P, and SN 2011ef) account for close to $50 \%$ of the total time covered by observations. Especially at large absolute magnitudes, the control-time distribution is dominated by the two nearby events, SN 2011dh and SN 2012P. In Figure 6, we show how the distribution changes when removing SN 2011dh, which owing to its small distance has a large contribution to the

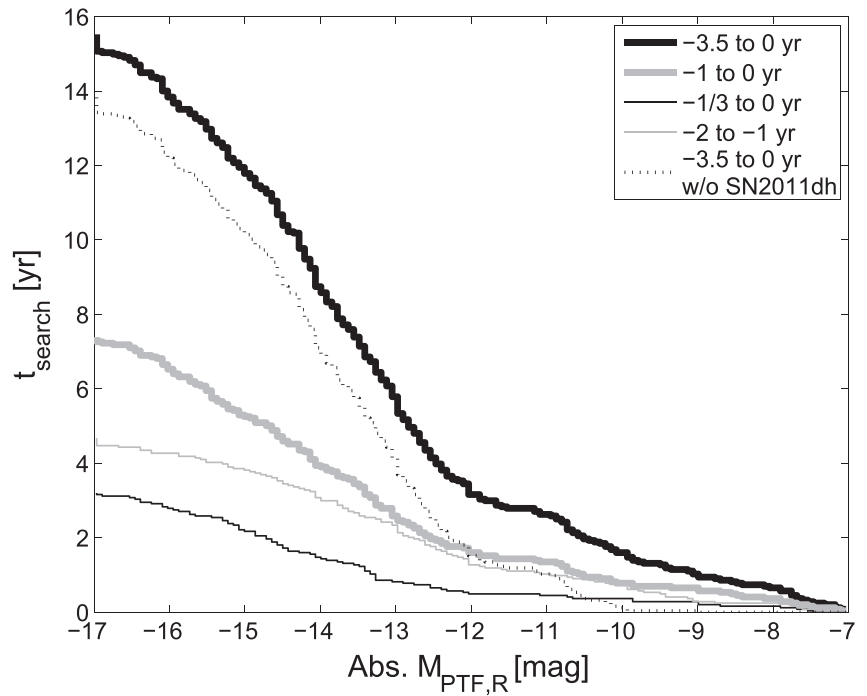

Figure 6. Absolute-magnitude-dependent control time for the complete sample, during which the SN locations were observed and no precursors were detected above the given magnitude. The various curves are for precursors taking place at different time ranges prior to the SN explosion (see the legend). The curve normalization and shape are determined by the amount of data and the SN distances. SN 2011dh accounts for most observations at the brightest magnitudes, and the dotted line shows the effect of removing it from the sample. Additional curves display the control time for shorter periods before the explosion. The limiting magnitudes per bin for all SNe are listed in Table 4.

control time in the absolute magnitude range of -7 to -10 . In a similar way, the second bump at magnitudes from -9 to -12 is due to the observations of SN 2012P. At lower magnitudes, many other SNe start to contribute to the distribution, so the results are less dominated by individual objects.

For searches in which no precursors are detected, we can set a $90 \%$ upper limit on the expectation number of precursors of 2.3 (Gehrels 1986; one-sided Poisson statistics). The limits on the average precursor rate per SN per year, $R_{\text {prec }}$, and on the average number of precursors per SN, $N_{\text {prec }}$, are given by

$$
R_{\text {prec }}<\frac{2.3}{t_{\text {search }}} \quad \text { and } \quad N_{\text {prec }}<2.3 \frac{t_{\text {window }}}{t_{\text {search }}},
$$

respectively, where $t_{\text {window }}$ is the duration of the considered time interval before the explosion. The limit on $N_{\text {prec }}$ is hence purely determined by the ratio $t_{\text {window }} / t_{\text {search }}$, the amount of 


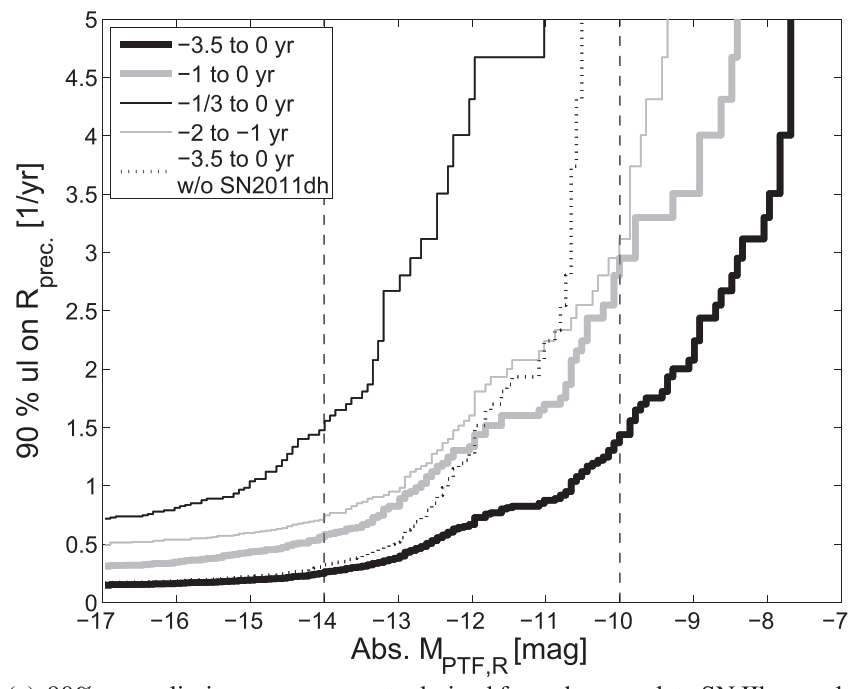

(a) $90 \%$ upper limit on precursor rate derived from the complete $\mathrm{SN}$ IIb sample

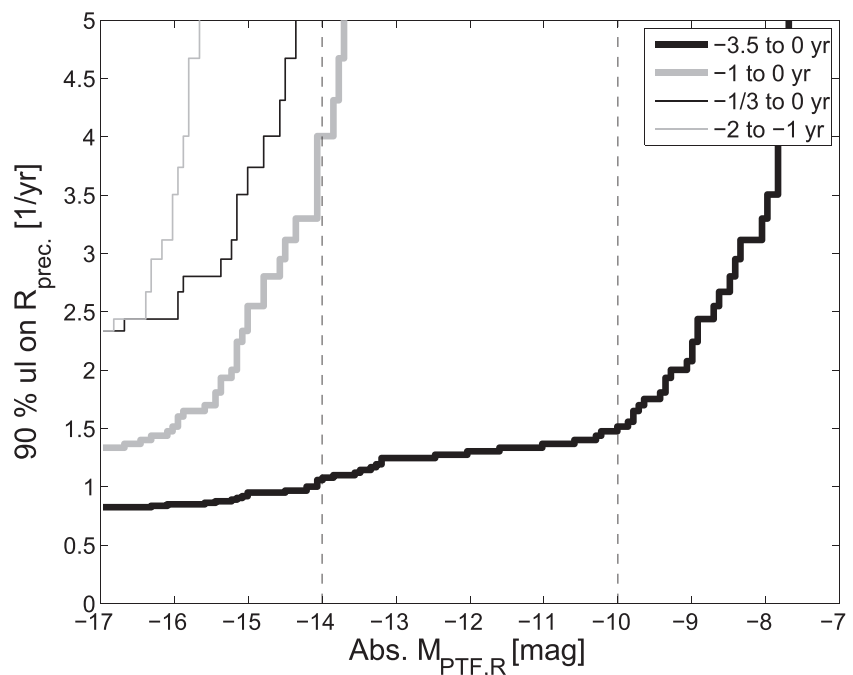

(b) $90 \%$ upper limit on precursor rate derived from five double-peaked $\mathrm{SNe} \mathrm{IIb}$

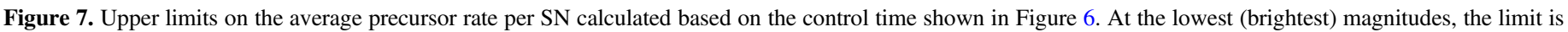
dominated by observations of SN 2011dh. Considering only double-peaked SNe, the limits are weaker owing to the smaller amount of data.

time during which precursors can be excluded compared to the time window during which the search is conducted. We note that $R_{\text {prec }}$ is an average quantity since the bins of $t_{\text {search }}$ are not necessarily distributed homogeneously within $t_{\text {window }}$.

The resulting upper limit on the precursor rate is shown in Figure 7(a), and Table 3 compares the upper limit on the number of precursors per SN to the number of precursors measured for SNe IIn by Ofek et al. (2014).

The upper limit on the number of precursors for the longest time interval of 3.5 years prior to the $\mathrm{SN}$ explosion is smaller than unity. This means that at the $90 \%$ confidence level, not all $\mathrm{SNe}$ IIb can exhibit precursors as bright as -14 mag, which is a typical $R$-band luminosity of precursors detected so far (e.g., Pastorello et al. 2007; Ofek et al. 2014). Compared to SNe IIn (Ofek et al. 2014), the average number of precursors prior to $\mathrm{SNe}$ IIb at magnitude -14 is lower by at least a factor of five (see Table 3). In case precursors are equally common before $\mathrm{SNe}$ IIb and SNe IIn, they have to be at least as dim as an absolute magnitude of -10 for $\mathrm{SNe} \mathrm{IIb}$, about a factor of 40 dimmer compared to the precursors observed prior to $\mathrm{SNe}$ IIn (see Table 3).

As explained in the Introduction, a double-peaked light curve presumably requires the presence of an extended hydrogen envelope (Bersten et al. 2012; Nakar \& Piro 2014). The duration of the first peak is usually only hours to a few days, and most $\mathrm{SNe}$ are not discovered as early. For most SNe in our sample, we thus do not know whether or not an early peak is present. However, not all SNe IIb have an early peak (I. Arcavi et al. 2015, in preparation), and there are indications that some SN IIb progenitors might be compact (Chevalier \& Soderberg 2010). To account for this diversity in the progenitor population, we additionally calculate limits considering only the data of the five SNe IIb for which two peaks are observed by PTF or reported in the literature. As stated in Table 1, this includes PTF 10tzh, SN 2011dh, PTF 12jaa, PTF 13ajn, and SN 2013df (see also I. Arcavi et al. 2015, in preparation). These SNe contribute only $15 \%$ of the control time of the complete sample, and the limits are hence drastically weakened, as shown in Figure 7(b).

\section{DISCUSSION}

In this section, we estimate whether or not the explosive ejection of a low-mass stellar envelope in a precursor event is likely to be bright enough to be detectable in this analysis. We expect a radiatively efficient precursor eruption to radiate an amount of energy comparable to (or larger than) the binding energy of the ejected envelope, which is given by

$$
E_{\text {bind }} \approx G \frac{M_{\text {env }} M_{\text {core }}}{R_{\text {core }}}
$$

where $G$ is the gravitational constant, $M_{\text {env }}$ and $M_{\text {core }}$ are, respectively, the masses of the envelope and the core, and $R_{\text {core }}$ is the core radius above which the envelope is located prior to its ejection.

According to Nakar \& Piro (2014; see also Piro 2015), both $M_{\text {env }}$ and $R_{\text {core }}$ can be estimated from the shape of the first peak in the optical light curve. $M_{\text {env }}$ is determined by the time at which the bolometric light curve reaches the first peak $\left(t_{\mathrm{p}}\right)$, and $R_{\text {core }}$ can be derived from the minimal luminosity $L_{\text {min }}$ between the two peaks. $L_{\min }$ can be obtained relatively precisely whenever a first peak is observed. The rise time $t_{\mathrm{p}}$ is usually less well constrained since it can be as short as a day or several hours, and in most cases the data are not sufficient.

In our sample, the only double-peaked $\mathrm{SN}$ for which a good upper limit on $t_{\mathrm{p}}$ is available is SN 2011dh, where the maximum of the first peak was reached at most $15 \mathrm{hr}$ after the explosion (Arcavi et al. 2011). This limit refers to the peak in visible light. However, the bolometric peak probably takes place even earlier; hence, this limit holds. For SN 2011dh, numerical simulations by Bersten et al. (2012) suggest $M_{\text {core }} \approx 4 M_{\odot}, M_{\text {env }} \approx 0.003 M_{\odot}$, and $R_{\text {core }} \approx 5 \times 10^{11} \mathrm{~cm}$, comparable to the values estimated by Nakar \& Piro (2014). Using Equation (3), the binding energy is $E_{\text {bind }} \approx 8 \times 10^{45} \mathrm{erg}$. This result is an order-of-magnitude estimate since most quantities entering the calculation have large uncertainties of up to a factor of a few. The kinetic energy required to unbind the envelope relates to the bolometric energy released in the precursor event, $E_{\text {prec}}$, via an unknown radiation efficiency 
factor $\epsilon$ : $E_{\text {rad,prec }}=\epsilon E_{\text {bind }}$ (see E. O. Ofek et al. 2015, in preparation).

In the case of SN 2011dh, within two years prior to the SN explosion, $50 \%$ of the time is covered by observations with a limiting magnitude of -9 or less and gaps no longer than two weeks. This limits the precursor luminosity during this time to $L_{\text {prec }}<10^{39} \mathrm{erg} \mathrm{s}^{-1}$ (no bolometric correction applied). If the envelope of this progenitor star is unbound during our observations ( $50 \%$ probability), we can set the following limit on the radiative efficiency $\epsilon$, as a function of the precursor duration $\delta t$, where the emitted luminosity is assumed to be constant over the precursor duration:

$$
\epsilon=\frac{E_{\mathrm{rad}, \mathrm{prec}}}{E_{\mathrm{bind}}} \approx \frac{L_{\mathrm{prec}} \delta t}{E_{\mathrm{bind}}} \lesssim 0.16\left(\frac{\delta t}{15 \text { days }}\right)
$$

The unbinding event (if it ever existed) might be too faint to be detected if the efficiency $\epsilon$ is low or if the precursor lasts for several months or longer. Another possibility is that the envelope is ejected prior to the time interval probed in this analysis.

The limit on the precursor luminosity $L_{\text {prec }}$ is close to the Eddington luminosity for a $4 M_{\odot}$ progenitor. Thus, a continuum-driven wind (e.g., Shaviv 2001), which could in principle explain giant eruptions of luminous blue variable stars or SN IIn precursors, is likely ruled out as an explanation for the SN IIb envelope ejection. Stellar winds can, however, be driven by other mechanisms such as line absorption or absorption by dust, which do not require as high luminosities; see Langer (2012) for a review.

If the precursor candidate detected prior to SN 2012cs is real, it released an energy of $\sim 4 \times 10^{46} \mathrm{erg}$ (see Section 4.2). We do not have much information about the progenitor star; however, if it is similar to the progenitor of SN $2011 \mathrm{dh}$, the radiated energy is comparable to the required energy for the unbinding event. It is therefore possible that a stellar envelope was ejected in this event.

\section{SUMMARY}

We examine the pre-explosion light curves of 27 nearby $\mathrm{SNe}$ $\mathrm{IIb}$, searching for outbursts. One precursor candidate is marginally detected in a single 60-day bin in the pre-explosion light curve of the nearby SN 2012cs. The probability to measure such an event caused by noise is $1.8 \%$ for this preexplosion light curve. The possible precursor happened 680 days before the SN explosion, and if real, its absolute $R$-band magnitude is $M_{R, \mathrm{PTF}}=-11.0$, which corresponds to a luminosity of $7 \times 10^{39} \mathrm{erg} \mathrm{s}^{-1}$ (no bolometric correction applied) and a radiative energy release of $4 \times 10^{46} \mathrm{erg}$ within the approximate duration of 60 days.

When binning the observations in 15-day bins, no precursor eruptions are found, and we calculate a magnitude-dependent limit on the average precursor rate among SNe IIb. Precursors as luminous as -14 mag occur on average $<0.86$ times within the final 3.5 years before the SN explosion, while in the last year the average number of precursors is limited to $<0.56$ at the $90 \%$ confidence level. These limits are obtained under the assumption that precursor eruptions last for at least 15 days. We conclude that bright precursor explosions, if they occur at all, are rare and do not happen before the explosion of every SN IIb.

By contrast, precursors are frequent for SNe IIn (Ofek et al. 2014 ); at the $99 \%$ confidence level, $98 \%$ of all SNe IIn have precursor eruptions brighter than absolute magnitude -14 within the 2.5 years before the explosion. The precursor rate of SNe IIn measured by Ofek et al. (2014) exceeds the upper limit for SNe IIb by about a factor of five at an absolute magnitude of -14 or less. In addition, the precursor rate for $\mathrm{SNe} \mathrm{IIb}$ can be constrained at higher magnitudes of up to -7 .

For the nearby SN IIb SN 2012P, our observations show that the source detected in pre-explosion HST observations at the SN position (Van Dyk et al. 2012) is still present more than two years after the SN explosion and thus cannot be the progenitor star.

The progenitor of SN 2011dh, the closest SN in our sample, is clearly detected in coadded observations, and its mean $R$-band magnitude is $m_{\mathrm{PTF}, ~}=21.82 \pm 0.08 \mathrm{mag}$, consistent with archival HST observations. The possible slow fading reported by Szczygieł et al. (2012) is below the sensitivity threshold of our observations. However, with 373 observations over the last two years prior to the $\mathrm{SN}$ explosion, the progenitor is monitored nearly constantly, and no variability or precursors are detected. We argue that for this progenitor star, the ejection of the stellar envelope in a precursor event might be observable, except if the process is radiatively inefficient or lasts over several months.

This paper is based on observations obtained with the Samuel Oschin Telescope as part of the PTF, a scientific collaboration between the California Institute of Technology, Columbia University, Las Cumbres Observatory, the Lawrence Berkeley National Laboratory, the National Energy Research Scientific Computing Center, the University of Oxford, and the Weizmann Institute of Science. We are grateful for excellent staff assistance at Palomar and Lick Observatories. E.O.O. is incumbent of the Arye Dissentshik career development chair and is grateful for support by grants from the Willner Family Leadership Institute Ilan Gluzman (Secaucus, NJ), Israeli Ministry of Science, Israel Science Foundation, Minerva and the I-CORE Program of the Planning and Budgeting Committee and The Israel Science Foundation. A.G.-Y. is supported by the EU/FP7 via ERC grant No. 307260, the Quantum universe I-Core program by the Israeli Committee for planning and budgeting, and the ISF, Minerva and ISF grants, WIS-UK "making connections" and the Kimmel and ARCHES awards. M.S. acknowledges support from the Royal Society and EU/FP7- ERC grant No. [615929]. N.J.S. thanks the IBM Einstein Fellowship support by the Institute for Advanced Study, Princeton. A.V.F's research was made possible by National Science Foundation grant AST-1211916, the TABASGO Foundation, and the Christopher R. Redlich Fund. The Lick Observatory Supernova Search was conducted with the Katzman Automatic Imaging Telescope, made possible by donations from Sun Microsystems, Inc., the Hewlett-Packard Company, AutoScope Corporation, Lick Observatory, the NSF, the University of California, the Sylvia \& Jim Katzman Foundation, and the TABASGO Foundation. Research at Lick Observatory is partially supported by a generous gift from Google.

\section{APPENDIX}

Representative spectra of all SNe discovered by PTF are shown in Figures 8-10 and are electronically available from the WISeREP webpage ${ }^{16}$ (Yaron \& Gal-Yam 2012). The spectra were acquired using various facilities that are listed in Table 5.

\footnotetext{
16 http://www.weizmann.ac.il/astrophysics/wiserep
} 


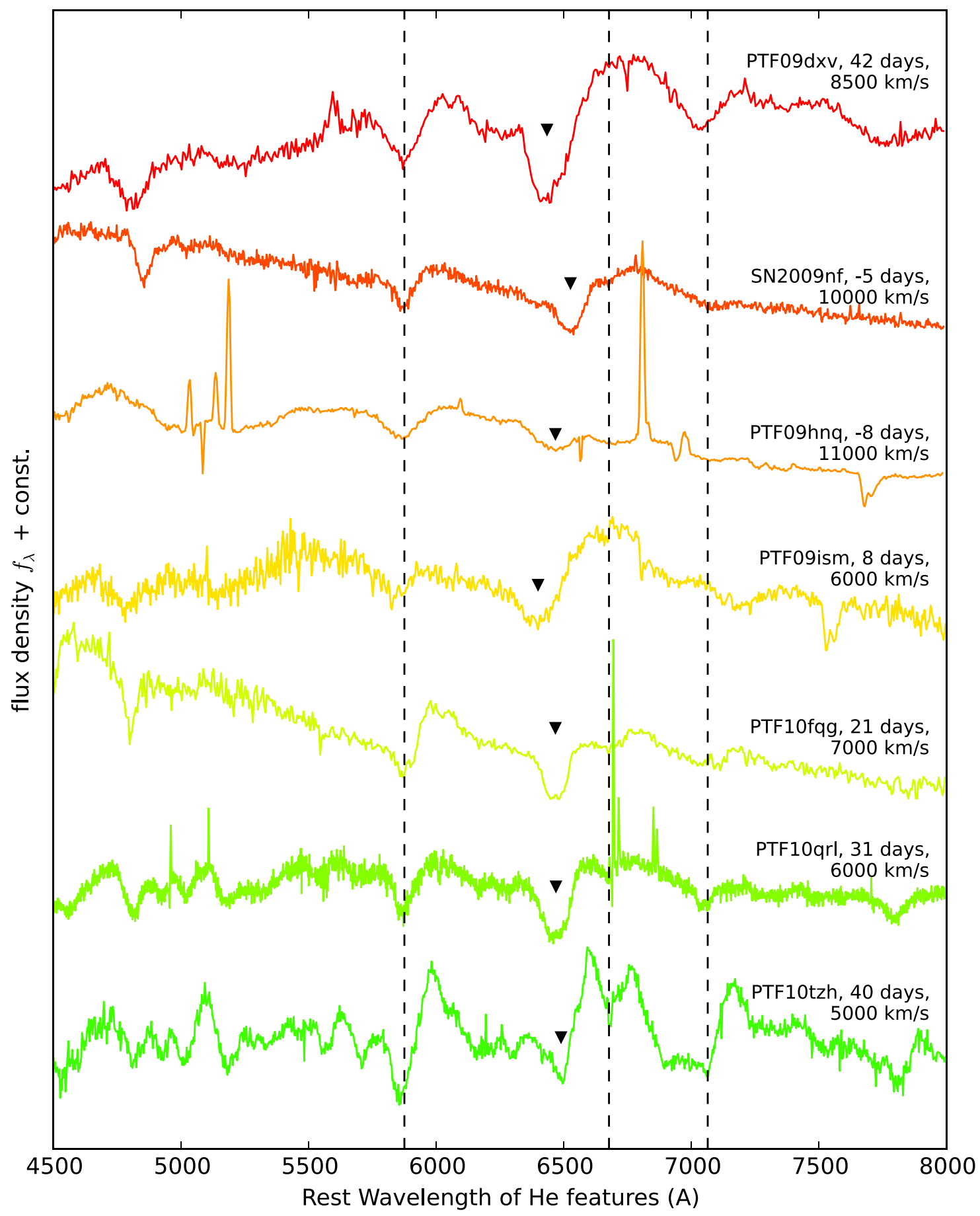

Figure 8. Representative spectra of the PTF-detected SNe in our sample. All spectra are redshifted by the indicated velocity relative to the rest frame such that their characteristic helium absorption features are at their rest wavelengths at 5876, 6678, and $7065 \AA$ (indicated by broken lines). In addition, the black triangles mark the prominent $\mathrm{H} \alpha$ absorption lines. The $\mathrm{H} \alpha$ emission line is found to the right of these features and often has a flat-top profile owing to the helium absorption at $6678 \AA$. Some SNe are compared to known SNe IIb identified with SNID (Blondin \& Tonry 2007) and the time after peak is indicated next to all spectra. All spectra are available electronically via the WISeREP webpage (Yaron \& Gal-Yam 2012). Further spectra are shown in Figures 9 and 10. 


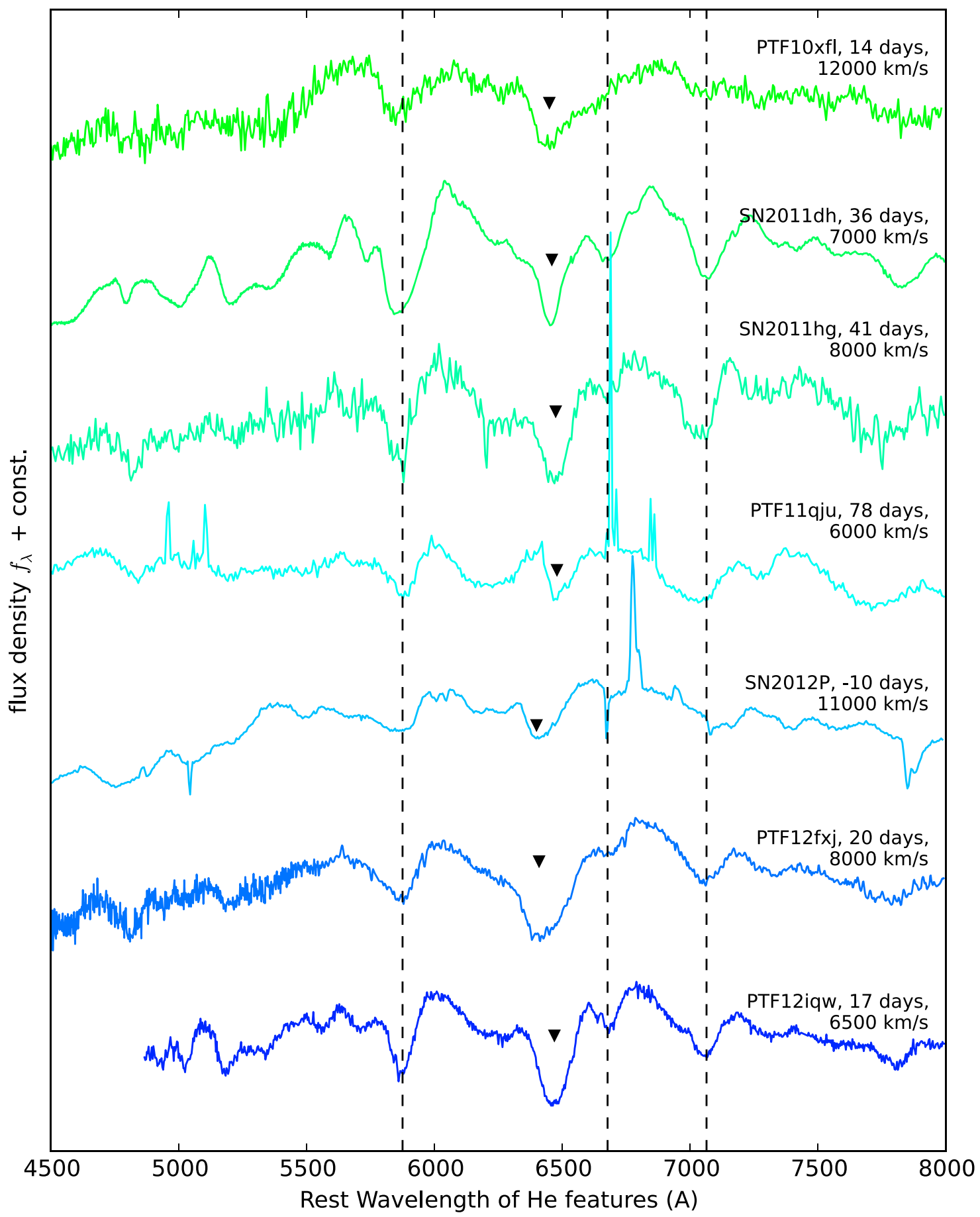

Figure 9. Figure 8, continued. 


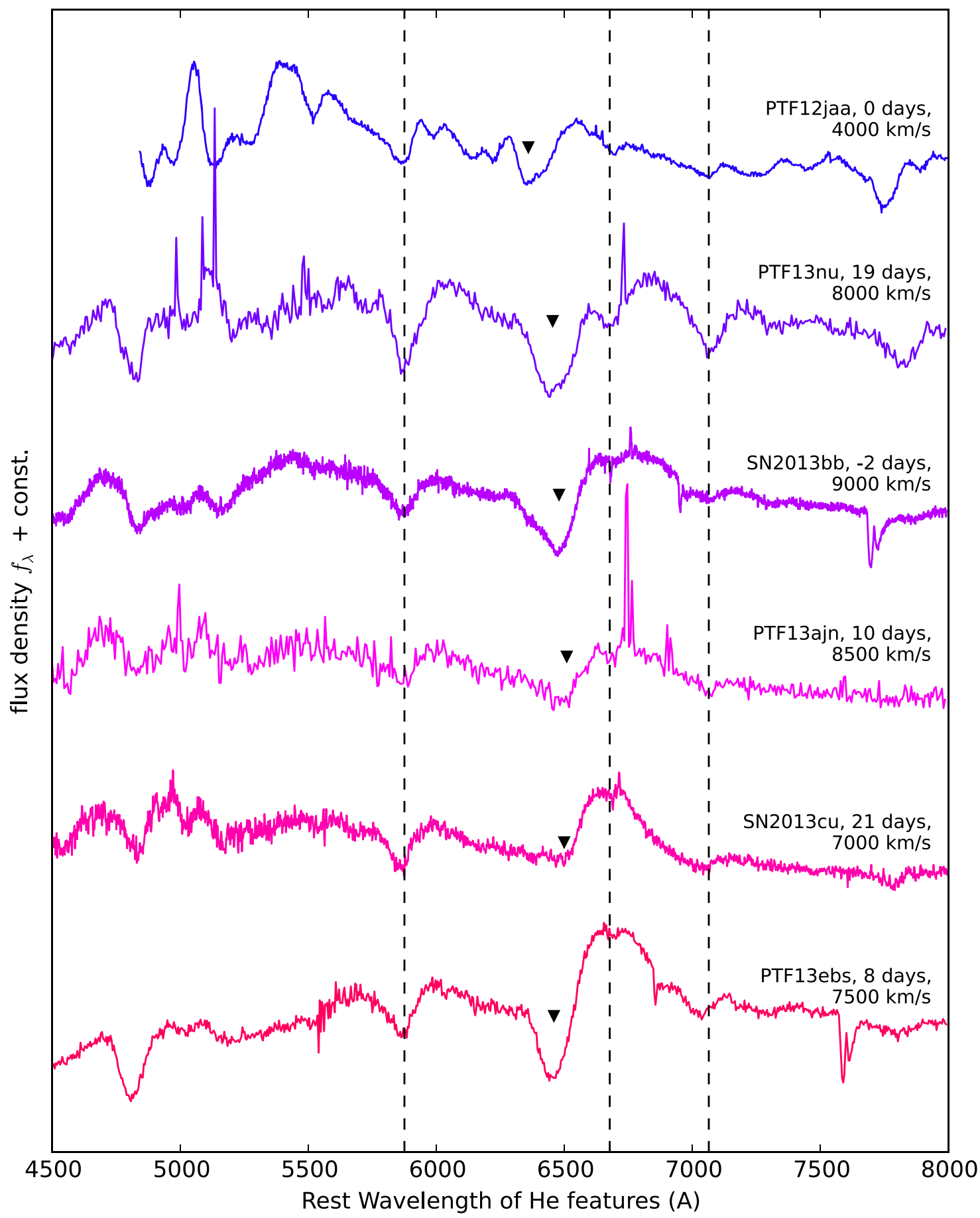

Figure 10. Figures 8 and 9, continued. 
Table 5

Log of Spectroscopic Observations

\begin{tabular}{lccc}
\hline \hline Name & Telescope & Instrument & Date \\
\hline PTF 09dxv & Keck I & LRIS & 2009 Oct 22 \\
SN 2009nf & Keck I & LRIS & 2009 Nov 11 \\
PTF 09hnq & HET & LRS & 2009 Nov 25 \\
PTF 09ism & P200 & DBSP & 2010 Jan 09 \\
PTF 10fqg & P200 & DBSP & 2010 May 07 \\
PTF 10qrl & Keck I & LRIS & 2010 Sep 05 \\
PTF 10tzh & Keck II & DEIMOS & 2010 Oct 12 \\
PTF 10xfl & P200 & DBSP & 2010 Oct 17 \\
SN 2011dh & Lick 3 m & Kast & 2011 Jul 06 \\
SN 2011hg & Lick 3 m & Kast & 2011 Dec 19 \\
PTF 11qju & Keck I & LRIS & 2012 Feb 20 \\
SN 2012P & HET & LRS & 2012 Jan 14 \\
PTF 12fxj & Lick 3 m & Kast & 2012 Jul 11 \\
SN 2012ey & Keck II & DEIMOS & 2012 Oct 14 \\
PTF 12jaa & Keck II & DEIMOS & 2012 Oct 14 \\
PTF 13nu & P200 & DBSP & 2013 Apr 13 \\
SN 2013bb & Keck I & LRIS & 2013 Apr 09 \\
PTF 13ajn & P200 & DBSP & 2013 May 02 \\
SN 2013cu & P200 & DBSP & 2013 Jun 02 \\
PTF 13ebs & Keck I & LRIS & 2013 Dec 02 \\
\hline
\end{tabular}

Note. The spectra are shown in Figures $8-10$.

\section{REFERENCES}

Alard, C., \& Lupton, R. H. 1998, ApJ, 503, 325

Arcavi, I., Gal-Yam, A., Ben-Ami, S., et al. 2012, ATel, 3881, 1

Arcavi, I., Gal-Yam, A., Kasliwal, M. M., et al. 2010, ApJ, 721, 777

Arcavi, I., Gal-Yam, A., Yaron, O., et al. 2011, ApJL, 742, L18

Arnett, W. D., \& Meakin, C. 2011a, ApJ, 733, 78

Arnett, W. D., \& Meakin, C. 2011b, ApJ, 741, 33

Baltay, C., Rabinowitz, D., Hadjiyska, E., et al. 2013, PASP, 125, 683

Ben-Ami, S., Hachinger, S., Gal-Yam, A., et al. 2015, ApJ, 803, 40

Benvenuto, O. G., Bersten, M. S., \& Nomoto, K. 2013, ApJ, 762, 74

Bersten, M. C., Benvenuto, O. G., Nomoto, K., et al. 2012, ApJ, 757, 31

Bilinski, C., Smith, N., Williams, G. G., et al. 2015, arXiv:1503.04252

Blanchard, P., Cenko, S. B., Li, W., et al. 2011, Central Bureau Telegram, 2772, 1

Blondin, S., \& Tonry, J. L. 2007, ApJ, 666, 1024

Borsato, L., \& Nascimbeni, V. 2012, CBET, 2993, 1

Bramich, D. M. 2008, MNRAS, 386, L77

Campbell, H., Blagorodnova, N., Fraser, M., et al. 2014, ATel, 5937, 1

Cardelli, J. A., Clayton, G. C., Mathis, J. S., et al. 1989, ApJ, 345, 245

Chen, T.-W., Fraser, M., \& Wright, D. 2012, CBET, 3043, 1

Chevalier, R. A. 2012, ApJL, 752, L2

Chevalier, R. A., \& Soderberg, A. M. 2010, ApJL, 711, L40

Chornock, R., Filippenko, A. V., Li, W., et al. 2011, ApJ, 739, 41

Ciabattari, F., \& Mazzoni, E. 2011, CBET, 2887, 1

Ciabattari, F., Mazzoni, E., Donati, S., et al. 2013, CBET, 3557, 1

Corsi, A., Ofek, E. O., Gal-Yam, A., et al. 2014, ApJ, 782, 42

Dimai, A., Briganti, F., \& Brimacombe, J. 2012, CBET, 2993, 1

Drake, A. J., Djorgovski, S. G., Mahabal, A., et al. 2009a, ApJ, 696, 870

Drake, A. J., Mahabal, A., Djorgovski, S. G., et al. 2009b, CBET, 2101, 1

Efron, B. 1982, The Jackknife, the Bootstrap and Other Resampling Plans (Philadelphia: SIAM)

Elias-Rosa, N., Taubenberger, S., Hachinger, S., et al. 2013, ATel, 4957, 1

Ergon, M., Jerkstrant, A., Sollerman, J., et al. 2014b, arXiv:1408.0731

Ergon, M., Sollerman, J., Fraser, M., et al. 2014a, A\&A, 562, A17
Filippenko, A. V. 1988, AJ, 96, 1941

Filippenko, A. V. 1997, ARAA, 35, 309

Filippenko, A. V., Li, W. D., Treffers, R. R., \& Modjaz, M. 2001, in ASP Conf. Ser. 246, Small-Telescope Astronomy on Global Scales, ed. W. P. Chen, C. Lemme \& B. Paczyński (San Francisco, CA: ASP), 121

Filippenko, A. V., Matheson, T., \& Barth, A. J. 1994, AJ, 108, 2220

Filippenko, A. V., Matheson, T., \& Ho, L. C. 1993, ApJL, 415, L103

Folatelli, G., Bersten, M. C., Benvenuto, O. G., et al. 2014, ApJL, 793, L22

Foley, R. J., Smith, N., Ganeshalingam, M., et al. 2007, ApJL, 657, L105

Fraser, M., Magee, M., Kotak, R., et al. 2013, ApJL, 779, L8

Gal-Yam, A., Arcavi, I., Ofek, E. O., et al. 2014, Natur, 509, 471

Gal-Yam, A., Ben-Ami, S., Yaron, O., et al. 2011, ATel, 3739, 1

Gehrels, N. 1986, ApJ, 303, 336

Griga, T., Marulla, A., Grenier, A., et al. 2011, CBET, 2736, 1

Hadjiyska, E., Walker, E., Rabinowitz, D., et al. 2012, ATel, 4563, 1

Hinshaw, G., Larson, D., Komatsu, E., et al. 2013, ApJS, 208, 19

Hodapp, K. W., Kaiser, N., Aussel, H., et al. 2004, AN, 325, 636

Horesh, A., Stockdale, C., Fox, D. B., et al. 2013, MNRAS, 436, 1258

Howell, D. A., Sullivan, M., Perrett, K., et al. 2005, ApJ, 634, 1190

Howerton, S., Drake, A. J., Djorgovski, S. G., et al. 2012, CBET, 3235, 1

Howerton, S., Drake, A. J., Djorgovski, S. G., et al. 2013, CBET, 3466, 1

Jha, S. W., McCully, C., \& Patel, B. 2012, ATel, 3035, 1

Laher, R. R., Surace, J., Grillmair, C. J., et al. 2014, PASP, 126, 674

Langer, N. 2012, ARA\&A, 50, 107

Law, N. M., Kulkarni, S. R., Dekany, R. G., et al. 2009, PASP, 121, 1395

Le Guillou, L., Baumont, S., Sullivan, M., et al. 2012, ATel, 4673, 1

Li, W. D., Filippenko, A. V., Treffers, R. R., et al. 2000, in AIP Conf. Ser. 522, Cosmic Explosions: Tenth Astrophysical Conference, ed. S. S. Holt \& W. W. Zhang (New York: AIP), 103

Li, W. D., Filippenko, A. V., \& van Dyk, S. D. 2011, ATel, 3401, 1

Lupton, R. H., Gunn, J. E., \& Szalav, A. S. 1999, AJ, 118, 1406

Marion, G. H., \& Berlind, P. 2011, CBET, 2894, 1

Marion, G. H., Vinko, G. H., Kirshner, R. P., et al. 2014, ApJ, 781, 69

Mauerhan, J. C., Smith, N., Filippenko, A. V., et al. 2013, MNRAS, 430, 1801

Maund, J. R., Fraser, M., Ergon, M., et al. 2011, ApJL, 739, L37

Morales-Garoffolo, A., Elias-Rosa, N., Benetti, S., et al. 2014, MNRAS, 445, 1647

Nakar, E., \& Piro, A. L. 2014, ApJ, 788, 193

Newton, J., Puckett, T., Lupi, F., et al. 2012, CBET, 3035, 1

Ofek, E. O. 2014, Astrophysics Source Code Library, record, ascl:1407.005

Ofek, E. O., Laher, R., Law, N., et al. 2012a, PASP, 124, 62

Ofek, E. O., Laher, R., Surace, J., et al. 2012b, PASP, 124, 854

Ofek, E. O., Sullivan, M., Cenko, S. B., et al. 2013, Natur, 494, 65

Ofek, E. O., Sullivan, M., Shaviv, N. J., et al. 2014, ApJ, 789, 104

Parrent, J., Howell, D. A., Nugent, R. C., et al. 2011, CBET, 2772, 1

Pastorello, A., Smartt, S. J., Mattila, S., et al. 2007, Natur, 447, 829

Piro, A. L. 2015, arXiv:1505.07103

Quataert, E., \& Shiode, J. 2012, MNRAS, 423, L92

Rakavy, G., \& Shaviv, G. 1967, ApJ, 148, 803

Rau, A., Kulkarni, S. R., Law, N. M., et al. 2009, PASP, 121, 1334

Rich, D., Elenin, L., \& Molotov, I. 2012, CBET, 3143, 1

Schlegel, D. J., Finkbeiner, D. P., \& Davis, M. 1998, ApJ, 500, 525

Shaviv, N. J. 2001, MNRAS, 326, 126

Shiode, J. H., \& Quataert, E. 2014, ApJ, 780, 96

Szczygieł, D. M., Gerke, J. R., Kochanek, C. S., \& Stanek, K. Z. 2012, ApJ, 747,23

Tomasella, L., Pastorello, A., Valenti, S., et al. 2011, CBET, 2887, 1

Tsvetkov, D. Y., Volkov, I. M., Baklanov, P., et al. 2009, PZ, 29, 2

Turatto, M., Pumi, M. L., Vanni, S., et al. 2012, ATel, 4386, 1

Van Dyk, S. D., Bradley, C., Foley, R. J., et al. 2013, ATel, 5139, 1

Van Dyk, S. D., Gal-Yam, A., Arcavi, I., et al. 2012, ATel, 3884, 1

Van Dyk, S. D., Weidong, L., Cenko, S. B., et al. 2011, ApJL, 741, L28

Van Dyk, S. D., Zheng, W., Fox, O. D., et al. 2014, AJ, 147, 37

Wheeler, J. C., Barker, E., Benjamin, R., et al. 1993, ApJL, 417, L71

Woosley, S. E., Eastman, R. G., Weaver, T. A., et al. 1994, ApJ, 429, 300

Yaron, O., \& Gal-Yam, A. 2012, PASP, 124, 668 